\title{
CONORMAL AND PIECEWISE SMOOTH SOLUTIONS TO QUASILINEAR WAVE EQUATIONS
}

\author{
SEONG JOO KANG
}

\begin{abstract}
In this paper, we show first that if a solution $u$ of the equation $P_{2}(t, x, u, D u, D) u=f(t, x, u, D u)$, where $P_{2}(t, x, u, D u, D)$ is a second order strictly hyperbolic quasilinear operator, is conormal with respect to a single characteristic hypersurface $\Sigma$ of $P_{2}$ in the past and $\Sigma$ is smooth in the past, then $\Sigma$ is smooth and $u$ is conormal with respect to $\Sigma$ for all time. Second, let $\Sigma_{0}$ and $\Sigma_{1}$ be characteristic hypersurfaces of $P_{2}$ which intersect transversally and let $\Gamma=\Sigma_{0} \cap \Sigma_{1}$. If $\Sigma_{0}$ and $\Sigma_{1}$ are smooth in the past and $u$ is conormal with repect to $\left\{\Sigma_{0}, \Sigma_{1}\right\}$ in the past, then $\Gamma$ is smooth, and $u$ is conormal with respect to $\left\{\Sigma_{0}, \Sigma_{1}\right\}$ locally in time outside of $\Gamma$, even though $\Sigma_{0}$ and $\Sigma_{1}$ are no longer necessarily smooth across $\Gamma$. Finally, we show that if $u(0, x)$ and $\partial_{t} u(0, x)$ are in an appropriate Sobolev space and are piecewise smooth outside of $\Gamma$, then $u$ is piecewise smooth locally in time outside of $\Sigma_{0} \cup \Sigma_{1}$.
\end{abstract}

\section{INTRODUCTION}

Let $u(t, x) \in C\left(\mathbf{R} ; H_{\mathrm{loc}}^{s}\left(\mathbf{R}^{n}\right)\right) \cap C^{1}\left(\mathbf{R} ; H_{\mathrm{loc}}^{s-1}\left(\mathbf{R}^{n}\right)\right), s>\frac{n}{2}+4$, be a solution of the quasilinear equation

$$
P_{2}(t, x, u, D u, D) u=f(t, x, u, D u),
$$

where

$$
P_{2}(t, x, u, D u, D) \equiv\left(\partial_{t}^{2}-\sum_{\substack{(i, j) \neq(0,0) \\ i, j=0}}^{n} a_{i j}(t, x, u, D u) \partial_{x_{i}} \partial_{x_{j}}\right)
$$

is strictly hyperbolic with respect to $\{t=$ constant $\}, f$ is a smooth function of its arguments and $\left(a_{i j}\right)$ is symmetric.

In this paper, we consider the regularity of solutions which are assumed to be conormal in the past with respect to a characteristic hypersurface, or a pair of characteristic hypersurfaces, or initial data conormal with respect to the intersection of a pair of characteristic hypersurfaces, and piecewise smoothness of solutions which are assumed to be piecewise smooth at $\{t=0\}$. The conormal cases for nonlinear problems had been treated previously by Alinhac [1] and [2]. He uses the theory of paradifferential operators and an extension to

Received by the editors May 17, 1993.

1991 Mathematics Subject Classification. Primary 35L70.

Key words and phrases. Strictly hyperbolic quasilinear equation, conormal, piecewise smooth. 
characteristic-coordinate changes. For the second order quasilinear case, he treated $H^{s}, s>(n+1) / 2+9 / 2$, conormal regularity with respect to a single characteristic hypersurface and $H^{s}, s>(n+1) / 2+5$, conormal regularity with respect to a pair of transverse characteristic hypersurfaces. Here we use nonsmooth vector fields in the given coordinates, with the regularity of the vector fields improved inductively, and the natural associated energy inequalities. Also we treat $C\left(\mathbf{R} ; H_{\mathrm{loc}}^{s}\left(\mathbf{R}^{n}\right)\right) \cap C^{1}\left(\mathbf{R} ; H_{\mathrm{loc}}^{s-1}\left(\mathbf{R}^{n}\right)\right), s>n / 2+4$, conormal regularity with respect to both a single characteristic hypersurface and a pair of transverse characteristic hypersurfaces. The piecewise smooth case for semilinear problems had been considered by Rauch and Reed [15].

In section 1, we prove first a version of the Gagliardo-Nirenberg inequalities, which takes an important role in the proofs of various lemmas and theorems. Then we establish energy inequalities for the equations $P_{2} u=f(D u)$ and $P_{2} v=$ $f_{1} D v+f_{2}$, where $f_{1}$ and $f_{2}$ are in the space $C\left(\mathbf{R} ; H_{\mathrm{loc}}^{s-5}\left(\mathbf{R}^{n}\right)\right)$. Also we consider the first order nonlinear equation satisfied by the defining function $\varphi\left(t, x^{\prime}\right)$ of a characteristic hypersurface of $P_{2}$. In order to treat this equation, we study the regularity of compositions of the form $v\left(t, \varphi\left(t, x^{\prime}\right), x^{\prime}\right)$ with $v\left(t, x_{1}, x^{\prime}\right)$ and $\varphi\left(t, x^{\prime}\right)$ assumed to have limited Sobolev regularity.

In section 2 , we prove first that if $u(t, x)$ is conormal with respect to a single characteristic hypersurface $\Sigma$ (which is locally given by $\left\{x_{1}=\varphi\left(t, x^{\prime}\right)\right\}$ ) of $P_{2}$ in the past and $\Sigma$ is smooth in the past, then $\Sigma$ is smooth and $u$ is conormal with respect to $\Sigma$ for all time. Analogous results hold locally in time, subject to the constraints of finite propagation speed. In order to prove conormal regularity, commutator arguments along with the energy inequality and the result on the regularity of compositions are then applied. Next, we consider the conormal regularity of $u$ with respect to a pair of transverse characteristic hypersurfaces of $P_{2}$. Let $\Sigma_{0}$ and $\Sigma_{1}$ be characteristic hypersurfaces of $P_{2}$ which intersect transversally and let $\Gamma=\Sigma_{0} \cap \Sigma_{1}$. If $\Sigma_{0}$ and $\Sigma_{1}$ are smooth in the past and $u$ is conormal with respect to $\left\{\Sigma_{0}, \Sigma_{1}\right\}$ in the past, then $\Gamma$ is smooth, and $u$ is conormal with respect to $\left\{\Sigma_{0}, \Sigma_{1}\right\}$ locally in time outside of $\Gamma$, even though $\Sigma_{0}$ and $\Sigma_{1}$ are no longer necessarily smooth across $\Gamma$. Examples of surfaces which are not smooth across $\Gamma$ in one-space dimension are presented in Messer [9]. In proving this property, we consider appropriate regions near $\Gamma$, and apply commutator and induction arguments involving vector fields with nonsmooth coefficients as in the proof of Theorem 3.2, as well as finite propagation speed. Finally, we prove that if $u(0, x)$ and $u_{t}(0, x)$ are conormal with respect to $\Gamma$, then $\Sigma_{0}$ and $\Sigma_{1}$ are smooth and $u$ is conormal with respect to $\left\{\Sigma_{0}, \Sigma_{1}\right\}$ locally in the future. The proof is analogous to the case of two characteristic hypersurfaces.

In section 3, we prove the piecewise smoothness of the solution $u$ of (1.1) assuming piecewise smoothness initially. Then after a change of coordinates, we may write

$$
\begin{aligned}
P_{2} & =Q_{+} D_{0}+\cdots \\
& =Q_{-} D_{1}+\cdots,
\end{aligned}
$$

where $Q_{+}$and $Q_{-}$are tangential vector fields to $\Sigma_{0}$ and $\Sigma_{1}$ respectively. Let $\mathscr{O}$ be an open neighborhood of the origin and let $\mathscr{O}_{0}=\mathscr{O} \cap\{t=0\}$. Let $\Sigma_{0}, \Sigma_{1}$ and $\Gamma$ be as above. With a slightly changed time coordinate, we may assume, by strict hyperbolicity of $P_{2}$ and the hypothesis of Theorem 3.8 
since $\Gamma$ is smooth, that $\Gamma$ divides $\mathscr{O}_{0}$ into two connected components and $\Sigma_{0} \cup \Sigma_{1}$ divides $\mathscr{O}$ in four connected components. Suppose that $u(0, x) \in$ $H_{\text {loc }}^{s}\left(\mathbf{R}^{n}\right)$ and $u_{t}(0, x) \in H_{\text {loc }}^{s-1}\left(\mathbf{R}^{n}\right)$ and both are piecewise smooth outside of $\Gamma$. Then $u$ is piecewise smooth locally in time. The proof involves Theorem 3.8 and repeated induction arguments together with a modification of the result of Rauch-Reed in the semilinear case: Suppose that $A(t, x)$ and $F(t, x)$ are piecewise continuous outside of $\Sigma_{0} \cup \Sigma_{1}$. Then the initial value problem for the linear system

$$
Q_{+} z+A z=F
$$

has a unique piecewise continuous solution on $\Sigma_{0}$ if $z(0, x)$ is piecewise continuous outside of $\Gamma$.

\section{Preliminaries}

2.1. The energy inequality. In this section we will prove appropriate energy inequalities for equation (1.1). We note that strictly hyperbolicity of $P_{2}(t, x, u, D u, D)$ implies the following inequality :

$$
|\xi|^{2} \leq C\left(\xi_{0}^{2}+\sum_{i, j=1}^{n} a_{i j}(t, x, u, D u) \xi_{i} \xi_{j}\right)
$$

where $C$ is a positive constant and $\xi=\left(\xi_{0}, \xi_{1}, \cdots, \xi_{n}\right) \in \mathbf{R}^{n+1}$. Throughout this thesis, we use

$$
\sum_{(i, j) \neq(0,0)}^{n} \text { instead of } \sum_{\substack{(i, j) \neq(0,0) \\ i, j=0}}^{n} \text { for notational convenience. }
$$

Before we prove the energy inequalities we need the following lemmas. For the proof of Schauder's lemma,see Rauch [13], and for the Gagliardo-Nirenberg inequalities, see Nirenberg [11].

Lemma 2.1 (Schauder's lemma). If $u, v \in H^{s}\left(\mathbf{R}^{n}\right)$ and $s>\frac{n}{2}$, then $u v \in$ $H^{s}\left(\mathbf{R}^{n}\right)$ and $\|u v\|_{H^{s}} \leq C\|u\|_{H^{s}}\|v\|_{H^{s}}$.

Lemma 2.2 (Gagliardo-Nirenberg inequalities). Let $u \in L^{q} \quad\left(\mathbf{R}^{n}\right)$ and its derivative of order $m, D^{m} u$, belong to $L^{r}\left(\mathbf{R}^{n}\right), 1 \leq q, r \leq \infty$. For the derivatives $D^{j} u, 0 \leq j<m$, the following inequalities hold:

$$
\left\|D^{j} u\right\|_{p} \leq \text { constant }\left\|D^{m} u\right\|_{r}^{a}\|u\|_{q}^{1-a},
$$

where $1 / p=j / n+a(1 / r-m / n)+(1-a) 1 / q$, for all $a$ in the interval $j / m \leq$ $a \leq 1$ (the constants depending only on $n, m, j, q, r$ and $a$ ) with the following exceptional cases:

(1) If $j=0, r m<n, q=\infty$ then we make the additional assumption that either $u$ tends to 0 at infinity or $u \in L^{\tilde{q}}\left(\mathbf{R}^{n}\right)$ for some finite $\tilde{q}>0$.

(2) If $1<r<\infty$, and $m-j-n / r$ is a nonnegative integer then (2.2) holds only for a satisfying $j / m \leq a<1$.

Throughout this paper we treat the case in which the regularity indices $s$ and $s^{\prime}$ are integers; analogous results hold in the general case. 
Lemma 2.3. Let $0 \leq s^{\prime} \leq s$ and suppose that $w \in L^{\infty}\left(\mathbf{R}^{n}\right) \cap H^{s}\left(\mathbf{R}^{n}\right)$. Then for $|\alpha|=s^{\prime}$, it follows that $D^{\alpha} w \in L^{2 p}\left(\mathbf{R}^{n}\right)$ and

$$
\left\|D^{\alpha} w\right\|_{L^{2 p}} \leq C\left(\|w\|_{L^{\infty}}\right)^{1-\frac{1}{p}}\left(\|w\|_{H^{s}}\right)^{\frac{1}{p}},
$$

where $p=s / s^{\prime}$ and $C$ is a constant depending only on $s, s^{\prime}$ and $n$.

Proof. Let $D^{s^{\prime}}$ stands for any derivative of order $s^{\prime}$. For $s^{\prime}=s$, we have $p=1$ and since $w \in H^{s}\left(\mathbf{R}^{n}\right),\left\|D^{s} w\right\|_{L^{2}} \leq C\|w\|_{H^{s}}$. For $0 \leq s^{\prime}<s$, by Lemma 2.2 for $q=\infty, r=2$ and $m=s$,

$$
\left\|D^{s^{\prime}} w\right\|_{L^{2 p}} \leq C\left\|D^{s} w\right\|_{L^{2}}^{a}\|w\|_{L^{\infty}}^{1-a},
$$

where $C$ is a constant depending only on $s, s^{\prime}$ and $n$, also $a$ is such that

$$
\frac{1}{2 p}=\frac{s^{\prime}}{n}+a\left(\frac{1}{2}-\frac{s}{n}\right) \quad \text { with } \frac{s^{\prime}}{s} \leq a<1 .
$$

From (2.3), we have for $a=s^{\prime} / s=1 / p$,

$$
\left\|D^{s^{\prime}} w\right\|_{L^{2 p}} \leq C\left(\|w\|_{L^{\infty}}\right)^{1-\frac{1}{p}}\left(\|w\|_{H^{s}}\right)^{\frac{1}{p}} .
$$

Corollary 2.4. Suppose that $w \in L_{\mathrm{loc}}^{\infty}\left(\mathbf{R}^{n}\right) \cap H_{\mathrm{loc}}^{s}\left(\mathbf{R}^{n}\right)$ for $s \geq 0$. If $f \in C^{\infty}(\mathbf{R})$, then $f(w) \in H_{\mathrm{loc}}^{s}\left(\mathbf{R}^{n}\right)$.

Proof. If $|\beta|=s$, then by the chain rule and the Leibniz formula, $D^{\beta}(f(w))$ may be written as a sum of terms of the form $g_{\beta}(w)\left(D^{\alpha_{1}} w\right) \cdots\left(D^{\alpha_{m}} w\right)$ with $g_{\beta}$ smooth and $\alpha_{1}+\cdots+\alpha_{m}=\beta$. Since $g_{\beta}(w) \in L_{\mathrm{loc}}^{\infty}\left(\mathbf{R}^{n}\right)$ and $D^{\alpha_{k}} w \in$ $L_{\text {loc }}^{r}\left(\mathbf{R}^{n}\right)$, where $r=\frac{2 s}{\left|\alpha_{k}\right|}$, by Lemma 2.3, Hölder's inequality implies that the use of the chain rule was justified, and since $\left(\left|\alpha_{1}\right|+\cdots+\left|\alpha_{m}\right|\right) / 2 s=1 / 2$, $g_{\beta}(w)\left(D^{\alpha_{1}} w\right) \cdots\left(D^{\alpha_{m}} w\right) \in L_{\mathrm{loc}}^{2}\left(\mathbf{R}^{n}\right)$.

Now we state and prove the energy inequalities for equation (1.1).

Theorem 2.5 (Energy Inequality). Let $P_{2}(t, x, u, D u, D)$ be a partial differential operator of order 2 on $\mathbf{R}^{n+1}$, as in (1.2), strictly hyperbolic with respect to the planes $t=$ constant and let $u(t, x) \in C\left(\mathbf{R} ; H_{\mathrm{loc}}^{s}\left(\mathbf{R}^{n}\right)\right) \cap C^{1}\left(\mathbf{R} ; H_{\mathrm{loc}}^{s-1}\left(\mathbf{R}^{n}\right)\right)$, $s>\frac{n}{2}+2$, satisfy the equation

$$
P_{2}(t, x, u, D u, D) u(t, x)=f(t, x, u, D u),
$$

where $f$ is a smooth function of its arguments. If $u(0, x) \in H_{\mathrm{loc}}^{s}\left(\mathbf{R}^{n}\right)$ and $u_{t}(0, x) \in H_{\mathrm{loc}}^{s-1}\left(\mathbf{R}^{n}\right)$, then, for all $t, u(t, x) \in H_{\mathrm{loc}}^{s}\left(\mathbf{R}^{n}\right)$. Moreover, if $u$ has compact support in $x$ for each time $t$, then we have

$$
\|u(t, x)\|_{H^{s}\left(\mathbf{R}^{n}\right)} \leq C_{t}\left(\|u(0, x)\|_{H^{s}\left(\mathbf{R}^{n}\right)}+\left\|u_{t}(0, x)\right\|_{H^{s-1}\left(\mathbf{R}^{n}\right)}\right)
$$

for all $t$, where $C_{t}=C(t)$ is independent of $u$ and $f$.

Proof. By finite propagation speed and an analysis local in time, it may be assumed that $u$ has compact support in $x$ for each $t$. Let $|\alpha|=s-1$. We apply $D^{\alpha} \equiv \partial_{x_{1}}^{\alpha_{1}} \cdots \partial_{x_{n}}^{\alpha_{n}}$ to equation (2.4) obtaining an equation, by the Leibniz formula,

$$
P_{2}\left(D^{\alpha} u\right)=D^{\alpha} f+\sum_{\beta<\alpha(i, j) \neq(0,0)} \sum_{\beta}^{n} C_{\beta}\left(D^{\alpha-\beta} a_{i j}\right)\left(D^{\beta} u_{x_{i} x_{j}}\right) \equiv g
$$


where $C_{\beta}$ are constants depending only on multiindices $\beta$. The use of the Leibniz formula is justified below, using Lemma 2.3. Let $w(t, x)=D^{\alpha} u(t, x)$. Then (2.6) can be written as

$$
P_{2} w \equiv w_{t t}-\sum_{(i, j) \neq(0,0)}^{n} a_{i j}(D u) w_{x_{i} x_{j}}=g .
$$

Let $E(t)$ be the energy for equation (2.7) defined by

$$
E^{2}(t)=\int\left(w^{2}(t, x)+w_{t}^{2}(t, x)+\sum_{i, j=1}^{n} a_{i j}(D u) w_{x_{i}}(t, x) w_{x_{j}}(t, x)\right) d x
$$

By differentiating (2.8) with respect to $t$ and integrating by parts, we have

$$
\begin{aligned}
2 E(t) & \frac{d E(t)}{d t} \\
= & \int\left(2 w w_{t}+2 w_{t} w_{t t}+\sum_{i, j=1}^{n}\left(\frac{\partial a_{i j}}{\partial t}\right) w_{x_{i}} w_{x_{j}}+2 \sum_{i, j=1}^{n} a_{i j} w_{x_{i} t} w_{x_{j}}\right) d x \\
= & \int\left(2 w w_{t}+2 w_{t} w_{t t}-2 \sum_{i, j=1}^{n} a_{i j} w_{t} w_{x_{i} x_{j}}-2 \sum_{i, j=1}^{n}\left(\frac{\partial a_{i j}}{\partial x_{i}}\right) w_{x_{j}} w_{t}\right) d x \\
& +\sum_{i, j=1}^{n} \int\left(\frac{\partial a_{i j}}{\partial t}\right) w_{x_{i}} w_{x_{j}} d x \\
= & 2 \int w_{t}\left(w+w_{t t}-\sum_{(i, j) \neq(0,0)}^{n} a_{i j} w_{x_{i} x_{j}}\right) d x+4 \int \sum_{j=1}^{n} a_{0 j} w_{t x_{j}} w_{t} d x \\
& -2 \sum_{i, j=1}^{n} \int\left(\frac{\partial a_{i j}}{\partial x_{i}}\right) w_{x_{j}} w_{t} d x+\sum_{i, j=1}^{n} \int\left(\frac{\partial a_{i j}}{\partial t}\right) w_{x_{i}} w_{x_{j}} d x .
\end{aligned}
$$

We note that

$$
4 \int \sum_{j=1}^{n} a_{0 j} w_{t x_{j}} w_{t} d x=-4 \int \sum_{j=1}^{n}\left(\frac{\partial a_{0 j}}{\partial x_{j}}\right) w_{t} w_{t} d x-4 \int \sum_{j=1}^{n} a_{0 j} w_{t x_{j}} w_{t} d x
$$

so that

$$
4 \int \sum_{j=1}^{n} a_{0 j} w_{t x_{j}} w_{t} d x=-2 \int \sum_{j=1}^{n}\left(\frac{\partial a_{0 j}}{\partial x_{j}}\right) w_{t} w_{t} d x
$$

It follows from (2.7) that

$$
\begin{aligned}
E(t) \frac{d E(t)}{d t}= & \int w_{t}(w+g) d x-\sum_{i, j=1}^{n} \int\left(\frac{\partial a_{i j}}{\partial x_{j}}\right) w_{x_{j}} w_{t} d x \\
& +\frac{1}{2} \int \sum_{i, j=1}^{n}\left(\frac{\partial a_{i j}}{\partial t}\right) w_{x_{i}} w_{x_{j}} d x-\int \sum_{j=1}^{n}\left(\frac{\partial a_{0 j}}{\partial x_{j}}\right) w_{t} w_{t} d x .
\end{aligned}
$$

By letting $\xi=\nabla w=\left(w_{t}, w_{x_{1}}, \cdots, w_{x_{n}}\right)$ in (2.1), we have

$$
w_{t}^{2}+w_{x_{1}}^{2}+\cdots+w_{x_{n}}^{2} \leq C\left(w_{t}^{2}+\sum_{i, j=1}^{n} a_{i j}(D u) w_{x_{i}} w_{x_{j}}\right)
$$


and so, by $(2.8)$,

$$
\int\left(w_{t}^{2}+\sum_{i, j=1}^{n} a_{i j}(D u) w_{x_{i}} w_{x_{j}}\right) d x \leq C E^{2}(t) .
$$

Now we estimate the third term in (2.9). Hölder's and Schwarz's inequalities imply that

$$
\begin{aligned}
\sum_{i, j=1}^{n} \int\left(\frac{\partial a_{i j}(D u)}{\partial t}\right) w_{x_{i}} w_{x_{j}} d x & \leq \sum_{i, j=1}^{n} \int\left|\left(\frac{\partial a_{i j}(D u)}{\partial t}\right) w_{x_{i}} w_{x_{j}}\right| d x \\
& \leq \sum_{i, j=1}^{n}\left(\left\|\frac{\partial a_{i j}(D u)}{\partial t}\right\|_{L^{\infty}} \int\left|w_{x_{i}} w_{x_{j}}\right| d x\right) \\
& \leq C(t) \sum_{i, j=1}^{n}\left(\int w_{x_{i}}^{2} d x\right)^{\frac{1}{2}}\left(\int w_{x_{j}}^{2} d x\right)^{\frac{1}{2}} \\
& \leq C(t) E^{2}(t),
\end{aligned}
$$

since $D^{2} u(t, x) \in H^{s-2}\left(\mathbf{R}^{n}\right) \subset L^{\infty}\left(\mathbf{R}^{n}\right)$, for all $t$, and $s-2>\frac{n}{2}$. Similarly, for the second and fourth terms, we have

$$
\begin{aligned}
\sum_{i, j=1}^{n} \int\left(\frac{\partial a_{i j}(D u)}{\partial x_{j}}\right) w_{x_{j}} w_{t} d x & \leq \sum_{i, j=1}^{n}\left(\left\|\frac{\partial a_{i j}(D u)}{\partial x_{j}}\right\|_{L^{\infty}\left(\mathbf{R}^{n}\right)} \int\left|w_{x_{i}} w_{t}\right| d x\right) \\
& \leq C(t) \sum_{i=1}^{n}\left(\int w_{x_{i}}^{2} d x\right)^{\frac{1}{2}}\left(\int w_{t}^{2} d x\right)^{\frac{1}{2}} \\
& \leq C(t) E^{2}(t),
\end{aligned}
$$

and

$$
\begin{aligned}
\sum_{j=1}^{n} \int\left(\frac{\partial a_{0 j}(D u)}{\partial x_{j}}\right) w_{t} w_{t} d x & \leq \sum_{j=1}^{n}\left(\left\|\frac{\partial a_{0 j}(D u)}{\partial x_{j}}\right\|_{L^{\infty}\left(\mathbf{R}^{n}\right)} \int w_{t}^{2} d x\right) \\
& \leq C(t) E^{2}(t) .
\end{aligned}
$$

For the first term, Schwarz's inequality yields that

$$
\begin{aligned}
\int w_{t}(w+g) d x & =\int w_{t} w d x+\int\left(w_{t} g\right) d x \\
& \leq C(t) E^{2}(t)+\int\left|w_{t} g\right| d x
\end{aligned}
$$

It remains to estimate $\int\left|w_{t} g\right| d x$.

We will show that for $1 \leq l \leq s-1,\left(D^{l} a_{i j}(D u)\right)\left(D^{s+1-l} u\right) \in L^{2}\left(\mathbf{R}^{n}\right)$. By the chain rule and the Leibniz formula, $D^{l} a_{i j}(D u)$ may be written as a sum of terms of the form $b_{\gamma}(D u)\left(D^{\alpha_{1}}(D u)\right) \cdots\left(D^{\alpha_{m}}(D u)\right)$ with $b_{\gamma}$ smooth and $\left|\alpha_{1}\right|+\cdots+\left|\alpha_{m}\right|=l$, where $\left|\alpha_{k}\right| \geq 1$ for $k=1, \cdots, m$. Let $D^{2} u(t, x)=$ $v(t, x)$. Then $v \in L^{\infty}\left(\mathbf{R}^{n}\right) \cap H^{s-2}\left(\mathbf{R}^{n}\right)$ since $s-2>n / 2$, and $D^{l} a_{i j}(D u)$ is written as a sum of terms of the form $b_{\gamma}(D u)\left(D^{\beta_{1}} v\right) \cdots\left(D^{\beta_{m}} v\right)$ with $\left|\beta_{1}\right|+$ $\cdots+\left|\beta_{m}\right| \leq l-1$, where $\left|\beta_{k}\right| \geq 0$ for $k=1, \cdots, m$. As we see in the 
proof of Corollary 2.4, $b_{\gamma}(D u) \in L^{\infty}\left(\mathbf{R}^{n}\right)$ and $D^{\beta_{k}} v \in L^{p}\left(\mathbf{R}^{n}\right)$, where $p=$ $\frac{2(s-2)}{\mid \beta_{k} !}$, by Lemma 2.3. Thus Hölder's inequality implies that the use of the chain rule was justified, and since $\left(\left|\beta_{1}\right|+\cdots+\left|\beta_{m}\right|\right) / 2(s-2) \leq(l-1) / 2(s-2)$, $b_{\gamma}(D u)\left(D^{\beta_{1}} v\right) \cdots\left(D^{\beta_{m}} v\right) \in L^{q}\left(\mathbf{R}^{n}\right)$, where $q=\frac{2(s-2)}{l-1}$. Since $D^{s+1-l} u=$ $D^{s-1-l} v, D^{s+1-l} u \in L^{r}\left(\mathbf{R}^{n}\right)$, where $r=\frac{2(s-2)}{s-1-l}$, by Lemma 2.3. Therefore, by Hölder's inequality and Lemma 2.3, $\left(D^{l} a_{i j}(D u)\right)\left(D^{s+1-l} u\right) \in L^{2}\left(\mathbf{R}^{n}\right)$ and $\left\|\left(D^{l} a_{i j}(D u)\right)\left(D^{s+1-l} u\right)\right\|_{L^{2}} \leq\left\|D^{l} a_{i j}(D u)\right\|_{L^{\prime}}\left\|D^{s+1-l} u\right\|_{L^{q}} \leq C(t)\|v\|_{L^{\infty}}\|v\|_{H^{s-2}}$ since $1 / p+1 / q=1 / 2$, where $p=2(s-2) /(l-1)$ and $q=2(s-2) /(s-1-l)$. As we have seen in the proof of Corollary 2.4, $D^{\alpha}(f(D u))$ may be written as a sum of terms of the form $f_{\alpha}(D u)\left(D^{\alpha_{1}}(D u)\right) \cdots\left(D^{\alpha_{m}}(D u)\right)$ with $f_{\alpha}$ smooth, $\alpha_{1}+\cdots+\alpha_{m}=\alpha$ and $f_{\alpha}(D u)\left(D^{\alpha_{1}}(D u)\right) \cdots\left(D^{\alpha_{m}}(D u)\right) \in L^{2}\left(\mathbf{R}^{n}\right)$. Moreover, by Lemma $2.3,\left\|D^{\alpha}(f(D u))\right\|_{L^{2}} \leq C(t)\|D u\|_{H^{s-1}} \leq C(t)\|u\|_{H^{s}}$.

By Schwarz's inequality, Minkowski's inequality and the facts above,

$$
\begin{aligned}
\int\left(w_{t} g\right) d x= & \int w_{t}\left(D^{\alpha} f+\sum_{\beta<\alpha} \sum_{(i, j) \neq(0,0)}^{n} C_{\beta}\left(D^{\alpha-\beta} a_{i j}\right)\left(D^{\beta} u_{x_{i} x_{j}}\right)\right) d x \\
\leq & \left(\int w_{t}^{2} d x\right)^{\frac{1}{2}}\left(\int\left(D^{\alpha} f\right)^{2} d x\right)^{\frac{1}{2}} \\
& +\left(\int w_{t}^{2} d x\right)^{\frac{1}{2}}\left[\sum_{\beta<\alpha(i, j) \neq(0,0)} \sum_{\beta}^{n} C_{\beta}\left(\int\left(D^{\alpha-\beta} a_{i j}\right)^{2}\left(D^{\beta} u_{x_{i} x_{j}}\right)^{2} d x\right)^{\frac{1}{2}}\right] \\
\leq & C(t) E(t)\left(\left\|D^{\alpha} f\right\|_{L^{2}}+\|v\|_{L^{\infty}}\|v\|_{H^{s-2}}\right) \\
\leq & C(t) E(t)\left(\|u\|_{H^{s}}\right) \\
\leq & C(t) E^{2}(t),
\end{aligned}
$$

and so

$$
\int w_{t}(w+g) d x \leq C(t) E^{2}(t)
$$

From $(2.10),(2.11),(2.12),(2.14)$ and by dividing $(2.9)$ by $E(t)$, we have

$$
\frac{d E(t)}{d t} \leq C(t) E(t)
$$

By applying Gronwall's inequality to (2.15),

$$
E(t) \leq \tilde{C}(t) E(0), \quad \text { where } d \tilde{C}(t)=\exp \left(\int_{0}^{t} C(\lambda) d \lambda\right) .
$$

Thus, for given $t$,

$$
\|u(t, x)\|_{H^{s}} \leq C(t)\left(\|u(0, x)\|_{H^{s}}+\left\|u_{t}(0, x)\right\|_{H^{s-1}}\right) .
$$

From the theorem above, we have the following corollary which will be used in the proofs of Theorem 3.2 and Theorem 3.8.

Corollary 2.6. Let $P_{2}(t, x, u, D u, D)$ be the same as above and assume that $u(t, x) \in C\left(\mathbf{R} ; H_{\mathrm{loc}}^{s}\left(\mathbf{R}^{n}\right)\right) \cap C^{1}\left(\mathbf{R} ; H_{\mathrm{loc}}^{s-1}\left(\mathbf{R}^{n}\right)\right), s>\frac{n}{2}+4$. Let $v(t, x)$ satisfy the equation

$$
P_{2}(t, x, u, D u, D) v(t, x)=f_{1}(t, x) D v(t, x)+f_{2}(t, x),
$$


where $f_{1}(t, x), f_{2}(t, x) \in C\left(\mathbf{R} ; H_{\mathrm{loc}}^{s-5}\left(\mathbf{R}^{n}\right)\right)$. If $v(0, x) \in H_{\mathrm{loc}}^{s-4}\left(\mathbf{R}^{n}\right)$ and $v_{t}(0, x) \in H_{\mathrm{loc}}^{s-5}\left(\mathbf{R}^{n}\right)$, then $v(t, x) \in H_{\mathrm{loc}}^{s-4}\left(\mathbf{R}^{n}\right)$ for all $t$. Moreover, if $v$ has compact support in $x$ for each time $t$, then we have

$$
\|v(t, x)\|_{H^{s-4}} \leq C_{1}(t)\left(\|v(0, x)\|_{H^{s-4}}+\left\|v_{t}(0, x)\right\|_{H^{s-s}}\right)+C_{2}(t),
$$

for all $t$, where $C_{1}(t), C_{2}(t)$ are indepedent of $u$ and $v$.

Proof. By finite propagation speed and analysis local in time, it may assumed that $v$ has compact support in $x$ for each $t$. As we see in the proof of the energy inequality 2.5 , we have

$$
\begin{aligned}
P_{2}\left(D^{\alpha} v\right)= & D^{\alpha}\left(f_{1}(t, x)(D v)\right)+D^{\alpha}\left(f_{2}(t, x)\right) \\
& +\sum_{\beta<\alpha(i, j) \neq(0,0)} \sum_{\beta}^{n} C\left(D^{\alpha-\beta} a_{i j}(D u)\right)\left(D^{\beta} v_{x_{i} x_{j}}\right) \\
\equiv & \tilde{g},
\end{aligned}
$$

where $|\alpha|=s-5$. Let $w(t, x)=D^{\alpha} v(t, x)$. Then (2.19) becomes

$$
P_{2} w \equiv w_{t t}-\sum_{(i, j) \neq(0,0)}^{n} a_{i j}(D u) w_{x_{i} x_{j}}=\tilde{g} .
$$

Let $\tilde{E}(t)$ be the energy for equation (2.20) defined as in (2.8). We will establish an a priori estimate on $\tilde{E}(t)$. As we see in the proof of energy inequality 2.5 , all estimates except $\int\left|w_{t} \tilde{g}\right| d x$ are valid, since $s>\frac{n}{2}+4$. So it remains to estimate $\int\left|w_{t} \tilde{g}\right| d x$. First we show that, for $1 \leq l \leq s-5,\left(D^{l} a_{i j}(D u)\right)\left(D^{s+1-l} v\right) \in$ $L^{2}\left(\mathbf{R}^{n}\right)$. From the proof of energy inequality $2.5, D^{l} a_{i j}(D u) \in L^{2 p}\left(\mathbf{R}^{n}\right)$, where $p=(s-2) /(l-1)$ and $\left\|D^{l} a_{i j}(D u)\right\|_{L^{2 p}} \leq C(t)\|u\|_{H^{s}} \leq C(t)$. Since $v \in$ $L^{\infty}\left(\mathbf{R}^{n}\right) \cap H^{s-4}\left(\mathbf{R}^{n}\right), s>\frac{n}{2}+4, D^{s-3-l} v \in L^{2 q}\left(\mathbf{R}^{n}\right)$, where $q=(s-4) /(s-3-l)$ and $\left\|D^{s-3-l} v\right\|_{L^{2 q}} \leq C(t)\|v\|_{H^{s-4}}^{1-\frac{1}{q}} \leq C(t) \tilde{E}(t)$, by Lemma 2.3. Therefore, $\left(D^{l} a_{i j}(D u)\right)\left(D^{s+1-l} v\right) \in L^{2}\left(\mathbf{R}^{n}\right)$, since

$$
(l-1) / 2(s-2)+(s-3-l) / 2(s-4) \leq 1 / 2,
$$

and

$$
\begin{aligned}
\left\|\left(D^{l} a_{i j}(D u)\right)\left(D^{s-3-l} v\right)\right\|_{L^{2}} & \leq\left\|D^{l} a_{i j}(D u)\right\|_{L^{2 p}}\left\|D^{s-3-l} v\right\|_{L^{2 q}} \\
& \leq C(t) \tilde{E}(t) .
\end{aligned}
$$

Next we estimate $D^{\alpha}\left(f_{1}(t, x)(D v)\right)$. By the Leibniz formula, $D^{\alpha}\left(f_{1}(t, x)(D v)\right)$ may be written as a sum of terms of the form $\left(D^{\alpha_{1}} f_{1}\right)\left(D^{\alpha_{2}}(D v)\right)$ with $\alpha_{1}+\alpha_{2}=$ $\alpha$. By Schwarz's inequality and Minkowski's inequality, $D^{\alpha}\left(f_{1}(t, x)(D v)\right) \in$ $L^{2}\left(\mathbf{R}^{n}\right)$ and $\left\|D^{\alpha}\left(f_{1}(t, x)(D v)\right)\right\|_{L^{2}} \leq\left\|f_{1}\right\|_{H^{s-s}} \tilde{E}(t)$.

Therefore, by the facts above,

$$
\begin{aligned}
\int\left(w_{t} \tilde{g}\right) d x= & \int w_{t}\left[D^{\alpha}\left(f_{1}(t, x)(D v)\right)+D^{\alpha}\left(f_{2}(t, x)\right)\right] \\
& +\int w_{t}\left[\sum_{\beta<\alpha(i, j) \neq(0,0)} \sum_{\beta}^{n} C\left(D^{\alpha-\beta} a_{i j}(D u)\right)\left(D^{\beta} v_{x_{i} x_{j}}\right)\right] d x \\
\leq & C(t) \tilde{E}(t)\left(\left\|f_{1}\right\|_{H^{s-s}} \tilde{E}(t)+\left\|f_{2}\right\|_{H^{s-s}}+\tilde{E}(t)\right),
\end{aligned}
$$


and so

$$
\frac{d \tilde{E}(t)}{d t} \leq C(t)\left(\left\|f_{1}\right\|_{H^{s-s}} \tilde{E}(t)+\left\|f_{2}\right\|_{H^{s-s}}\right) .
$$

Therefore, by applying Gronwall's inequality to (2.21),

$$
\tilde{E}(t) \leq e^{h(t)}\left(\tilde{E}(0)+\int_{0}^{t} C(\tau)\left\|f_{2}(\tau)\right\|_{H^{s-s}} e^{-h(\tau)} d \tau\right),
$$

where

$$
h(t)=\int_{0}^{t} C(\tau)\left\|f_{1}(\tau)\right\|_{H^{s-s}} d \tau \quad \text { and } \quad f_{i}(\tau) \equiv f_{i}(\tau, x), \quad i=1,2 .
$$

Let $C_{1}(t)=e^{h(t)}$ and $C_{2}(t)=e^{h(t)} \int_{0}^{t} C(\tau)\left\|f_{2}(\tau)\right\|_{H^{s-s}} e^{-h(\tau)} d \tau$. Then we have

$$
\tilde{E}(t) \leq C_{1}(t) \tilde{E}(0)+C_{2}(t),
$$

which implies that $\tilde{E}(t)$ is finite for all time $t$, since by assumption it is finite at $t=0$. Therefore,

$$
\|v(t, x)\|_{H^{s-4}} \leq C_{1}(t)\left(\|v(0, x)\|_{H^{s-4}}+\left\|v_{t}(0, x)\right\|_{H^{s-s}}\right)+C_{2}(t) .
$$

2.2. First order nonlinear equations. Suppose that a characteristic hypersurface for $P_{2}$ is given by $\left\{x_{1}=\varphi\left(t, x^{\prime}\right)\right\}$, where $x^{\prime}=\left(x_{2}, \cdots, x_{n}\right) \in \mathbf{R}^{n-1}$. Then $\varphi$ satisfies the first order nonlinear equation

$$
\left(\varphi_{t}\right)^{2}+2\left(a_{01}-\sum_{j=2}^{n} a_{0 j} \varphi_{x_{j}}\right) \varphi_{t}=a_{11}-2 \sum_{j=2}^{n} a_{1 j} \varphi_{x_{j}}+\sum_{i, j=2}^{n} a_{i j} \varphi_{x_{i}} \varphi_{x_{j}} .
$$

The coefficients in (2.22) are evaluated at $x_{1}=\varphi\left(t, x^{\prime}\right)$, and thus the regularity of functions of the form $a\left(D u\left(t, \varphi\left(t, x^{\prime}\right), x^{\prime}\right)\right)$ needs to be examined.

We begin by studying the regularity of compositions of the form $v\left(\varphi\left(x^{\prime}\right), x^{\prime}\right)$ with $v$ and $\varphi$ assumed to have limited Sobolev regularity. Even if $\varphi\left(x^{\prime}\right)$ is smooth, functions of the form $v\left(\varphi\left(x^{\prime}\right), x^{\prime}\right)$ will in general have Sobolev regularity of order $1 / 2$ lower than that of $v\left(x_{1}, x^{\prime}\right)$. When $\varphi\left(x^{\prime}\right)$ is nonsmooth, the regularity of $v\left(\varphi\left(x^{\prime}\right), x^{\prime}\right)$ will not in general be greater than that of $\varphi\left(x^{\prime}\right)$. In order to obtain norm estimates on the regularity of $v\left(\varphi\left(x^{\prime}\right), x^{\prime}\right)$ that are linear in the norm of $\varphi\left(x^{\prime}\right)$, we will assume that the Sobolev regularity of $v\left(x_{1}, x^{\prime}\right)$ is at least one order greater than that of $\varphi\left(x^{\prime}\right)$.

Lemma 2.7. Let $v\left(x_{1}, x^{\prime}\right) \in H_{\mathrm{loc}}^{s+1}\left(\mathbf{R}^{n}\right)$ for $s>\frac{n}{2}+1$. Suppose that $\varphi\left(x^{\prime}\right) \in$ $H_{\text {loc }}^{s^{\prime}}\left(\mathbf{R}^{n-1}\right), 1 \leq s^{\prime} \leq s$, and that $D \varphi\left(x^{\prime}\right) \in L^{\infty}\left(\mathbf{R}^{n-1}\right)$. Then

$$
v\left(\varphi\left(x^{\prime}\right), x^{\prime}\right) \in H_{\text {loc }}^{s^{\prime}}\left(\mathbf{R}^{n-1}\right) .
$$

If $v$ and $\varphi$ have compact support, then $\left\|v\left(\varphi\left(x^{\prime}\right), x^{\prime}\right)\right\|_{H^{s^{\prime}}} \leq C\|\varphi\|_{H^{s^{\prime}}}$ with $C$ depending only on $s, s^{\prime}, n$, the size of the supports, $\left\|v\left(x_{1}, x^{\prime}\right)\right\|_{H^{s+1}}$ and $\|\varphi\|_{L \infty}$.

Proof. We may assume without loss of generality that $v$ and $\varphi$ have compact support. Let $w$ represent the vector consisting of all derivatives of $\partial_{x_{1}} v$ (i.e., $\left.w=\left(\partial_{x_{1}}\left(\partial_{x_{1}} v\right), \cdots, \partial_{x_{n}}\left(\partial_{x_{1}} v\right)\right)\right)$. Then $w \in H^{s-1}\left(\mathbf{R}^{n}\right) \cap L^{\infty}\left(\mathbf{R}^{n}\right)$ since $s-1>$ $\frac{n}{2}$, and therefore $D^{\alpha} w \in L^{r}\left(\mathbf{R}^{n}\right)$, where $r=\frac{2(s-1)}{|\alpha|}$, for $0 \leq|\alpha| \leq s-1$ by 
Lemma 2.3. Thus $D^{\beta} \partial_{x_{1}} v \in L^{r^{\prime}}\left(\mathbf{R}^{n}\right)$, where $r^{\prime}=\frac{2(s-1)}{|\beta|-1}$, for $1 \leq|\beta| \leq s$, and since

$$
\left(D^{\beta} v\right)\left(\varphi\left(x^{\prime}\right), x^{\prime}\right)=\int_{-\infty}^{\varphi\left(x^{\prime}\right)} D^{\beta} \partial_{x_{1}} v\left(x_{1}, x^{\prime}\right) d x_{1}
$$

it follows from Minkowski's inequality that $\left(D^{\beta} v\right)\left(\varphi\left(x^{\prime}\right), x^{\prime}\right) \in L^{r}\left(\mathbf{R}^{n-1}\right)$, where $r=\frac{2(s-1)}{|\beta|-1}$, with norm depending only on the size of the supports, $\left\|v\left(x_{1}, x^{\prime}\right)\right\|_{H^{s+1}}$ and $\|\varphi\|_{L^{\infty}}$.

Next, notice that $D \varphi \in H^{s^{\prime}-1}\left(\mathbf{R}^{n-1}\right) \cap L^{\infty}\left(\mathbf{R}^{n-1}\right)$, and therefore $D^{\beta} \varphi \in L^{r^{\prime}}$ $\left(\mathbf{R}^{n-1}\right)$, where $r^{\prime}=\frac{2\left(s^{\prime}-1\right)}{|\beta|-1}$, for $1 \leq|\beta| \leq s^{\prime}$, by Lemma 2.3. The chain rule and the Leibniz formula imply that, for $1 \leq|\gamma| \leq s^{\prime}, D^{\gamma}\left(v\left(\varphi\left(x^{\prime}\right), x^{\prime}\right)\right)$ may be written as a sum of terms of the form

$$
\left(D^{\beta_{0}} D^{m} v\right)\left(\varphi\left(x^{\prime}\right), x^{\prime}\right)\left(D^{\beta_{1}} \varphi\left(x^{\prime}\right)\right) \cdots\left(D^{\beta_{m}} \varphi\left(x^{\prime}\right)\right),
$$

where $D^{m}$ stands for a derivative of order $m$ with respect to $x_{1}$ with $0 \leq$ $m \leq s^{\prime}$ and $\beta_{0}+\beta_{1}+\cdots+\beta_{m}=\gamma, 1 \leq\left|\beta_{k}\right| \leq s^{\prime}$ for $k=0,1, \cdots, m$. If $m=0$, then $\gamma=\beta_{0}$, and so $\left(D^{\beta_{0}} v\right)\left(\varphi\left(x^{\prime}\right), x^{\prime}\right) \in L^{q}\left(\mathbf{R}^{n-1}\right) \subset L^{2}\left(\mathbf{R}^{n-1}\right)$, where $q=\frac{2(s-1)}{\left|\beta_{0}\right|-1}$, with $\left\|\left(D^{\beta_{0}} v\right)\left(\varphi\left(x^{\prime}, x^{\prime}\right)\right)\right\|_{L^{2}} \leq C$, where $C$ depends only on the size of supports, $s, s^{\prime}, n,\left\|v\left(x_{1}, x^{\prime}\right)\right\|_{H^{s+1}}$ and $\|\varphi\|_{L^{\infty}}$. Therefore, we may assume that $m \geq 1$. The preceding estimates and Hölder's inequality imply that the use of the chain rule was justified, and

$$
\left(D^{\beta_{0}} D^{m} v\right)\left(\varphi\left(x^{\prime}\right), x^{\prime}\right)\left(D^{\beta_{1}} \varphi\left(x^{\prime}\right)\right) \cdots\left(D^{\beta_{m}} \varphi\left(x^{\prime}\right)\right) \in L^{2}\left(\mathbf{R}^{n-1}\right)
$$

since

$$
\frac{\left|\beta_{0}\right|+(m-1)}{2(s-1)}+\frac{\left(\left|\beta_{1}\right|-1\right)+\cdots+\left(\left|\beta_{m}\right|-1\right)}{2\left(s^{\prime}-1\right)} \leq \frac{|\gamma|-1}{2\left(s^{\prime}-1\right)} \leq \frac{1}{2} .
$$

Therefore $D^{\gamma}\left(v\left(\varphi\left(x^{\prime}\right), x^{\prime}\right)\right) \in L^{2}\left(\mathbf{R}^{n-1}\right)$ for $1 \leq|\gamma| \leq s^{\prime}$. Moreover, by Lemma 2.3, $\left\|D^{\gamma}\left(v\left(\varphi\left(x^{\prime}\right), x^{\prime}\right)\right)\right\|_{L^{2}}$ is bounded up to an appropriate constant by

$$
\left(\|D \varphi\|_{H^{s^{\prime}-1}}\right)^{\frac{\left|\beta_{j}\right|-1}{s^{\prime}-1}} \cdots\left(\|D \varphi\|_{H^{s^{\prime}-1}}\right)^{\frac{\left|\beta_{m}\right|-1}{s^{\prime}-1}} .
$$

Since $\left(\left|\beta_{1}\right|-1\right)+\cdots+\left(\left|\beta_{m}\right|-1\right) \leq|\gamma|-m \leq s^{\prime}-m \leq s^{\prime}-1$, the required estimate holds.

For solutions of hyperbolic equations, it is natural to consider functions which are continuous in time, with values in appropriate Sobolev spaces in the remaining variables.

Corollary 2.8. Let $v\left(t, x_{1}, x^{\prime}\right) \in C\left(\mathbf{R} ; H_{\mathrm{loc}}^{s+1}\left(\mathbf{R}^{n}\right)\right)$ for $s>\frac{n}{2}+1$. Suppose $\varphi(t, x) \in C\left(\mathbf{R} ; H_{\mathrm{loc}}^{s^{\prime}}\left(\mathbf{R}^{n-1}\right)\right), 1 \leq s^{\prime} \leq s$, and that $D \varphi\left(t, x^{\prime}\right) \in C\left(\mathbf{R} ; L_{\mathrm{loc}}^{\infty}\left(\mathbf{R}^{n-1}\right)\right)$. Then $v\left(t, \varphi\left(t, x^{\prime}\right), x^{\prime}\right) \in C\left(\mathbf{R} ; H_{\mathrm{loc}}^{s^{\prime}}\left(\mathbf{R}^{n-1}\right)\right)$.

Proof. The proof of Lemma 2.7 easily yields that

$$
D^{\beta} \partial_{x_{1}} v\left(t, x_{1}, x^{\prime}\right) \in C\left(\mathbf{R} ; L^{\frac{2(s-1)}{|\beta|-1}}\left(\mathbf{R}^{n}\right)\right) \quad \text { for } 1 \leq|\beta| \leq s .
$$


Let $p=2(s-1) /(|\beta|-1)$. Then, from (2.23) with $t$ as a parameter,

$$
\begin{aligned}
& \left(D^{\beta} v\right)\left(t, \varphi\left(t, x^{\prime}\right), x^{\prime}\right)-\left(D^{\beta} v\right)\left(t, \varphi\left(t_{0}, x^{\prime}\right), x^{\prime}\right) \\
& \quad=\int_{-\infty}^{\varphi\left(t, x^{\prime}\right)} D^{\beta} \partial_{x_{1}} v\left(t, x_{1}, x^{\prime}\right) d x_{1}-\int_{-\infty}^{\varphi\left(t_{0}, x^{\prime}\right)} D^{\beta} \partial_{x_{1}} v\left(t, x_{1}, x^{\prime}\right) d x_{1} \\
& \quad=\int_{\varphi\left(t_{0}, x^{\prime}\right)}^{\varphi\left(t, x^{\prime}\right)} D^{\beta} \partial_{x_{1}} v\left(t, x_{1}, x^{\prime}\right) d x_{1} .
\end{aligned}
$$

It follows that

$$
\begin{aligned}
& \|\left(D^{\beta} v\right)\left(t, \varphi\left(t, x^{\prime}\right), x^{\prime}\right)-\left(D^{\beta} v\right)\left(t, \varphi\left(t_{0}, x^{\prime}\right), x^{\prime}\right) \|_{L^{p}\left(\mathbf{R}^{n-1}\right)} \\
& \leq C\left\|\varphi\left(t, x^{\prime}\right)-\varphi\left(t_{0}, x^{\prime}\right)\right\|_{L^{\infty}(\mathbf{R})} \rightarrow 0 \quad \text { as } t \rightarrow t_{0},
\end{aligned}
$$

and similarly

$$
\begin{aligned}
\left\|\left(D^{\beta} v\right)\left(t, \varphi\left(t_{0}, x^{\prime}\right), x^{\prime}\right)-\left(D^{\beta} v\right)\left(t_{0}, \varphi\left(t_{0}, x^{\prime}\right), x^{\prime}\right)\right\|_{L^{p}\left(\mathbf{R}^{n-1}\right)} \\
\leq C\left\|v\left(t, x_{1}, x^{\prime}\right)-v\left(t_{0}, x_{1}, x^{\prime}\right)\right\|_{H^{s+1}\left(\mathbf{R}^{n}\right)} \rightarrow 0 \quad \text { as } t \rightarrow t_{0} .
\end{aligned}
$$

Therefore

$$
\left(D^{\beta} v\right)\left(t, \varphi\left(t, x^{\prime}\right), x^{\prime}\right) \in C\left(\mathbf{R} ; L^{p}\left(\mathbf{R}^{n-1}\right)\right) \quad \text { for } 1 \leq|\beta| \leq s .
$$

We note that $D \varphi \in C\left(\mathbf{R} ; H^{s^{\prime}-1}\left(\mathbf{R}^{n-1}\right)\right) \cap C\left(\mathbf{R} ; L^{\infty}\left(\mathbf{R}^{n-1}\right)\right)$ and therefore

$$
D^{\beta} \varphi \in C\left(\mathbf{R} ; L^{\frac{2\left(s^{\prime}-1\right)}{|\beta|-1}}\left(\mathbf{R}^{n-1}\right)\right) \quad \text { for } 1 \leq|\beta| \leq s^{\prime},
$$

by Lemma 2.3. The rest of the proof of Lemma 2.7 then easily yields continuity of the norm estimates in the parameter $t$.

In the proof of conormal regularity of a solution of the equation $P_{2} u=$ $f(D u)$, where $f$ is smooth of its arguments, we will encounter situations in which regularity of $\varphi$ with respect to $v$ is greater than the case considered above.

Lemma 2.9. Let $v\left(x_{1}, x^{\prime}\right) \in H_{\mathrm{loc}}^{s+1}\left(\mathbf{R}^{n}\right)$ for $s>\frac{n}{2}$. Suppose that $\varphi\left(x^{\prime}\right) \in$ $H_{\mathrm{loc}}^{s+1}\left(\mathbf{R}^{n-1}\right)$ and that $D \varphi\left(x^{\prime}\right) \in L^{\infty}\left(\mathbf{R}^{n-1}\right)$. Then $v\left(\varphi\left(x^{\prime}\right), x^{\prime}\right) \in H_{\mathrm{loc}}^{s}\left(\mathbf{R}^{n-1}\right)$. If $v$ and $\varphi$ have compact support, then $\left\|v\left(\varphi\left(x^{\prime}\right), x^{\prime}\right)\right\|_{H^{s}} \leq C\|\varphi\|_{H^{s}}$ with $C$ depending only on $s, n$, the size of the supports, $\left\|v\left(x_{1}, x^{\prime}\right)\right\|_{H^{s+1}}$ and $\|\varphi\|_{L^{\infty}}$.

Proof. We may assume without loss of generality that $v$ and $\varphi$ have compact support. Since $s>\frac{n}{2}, \partial_{x_{1}} v \in H^{s}\left(\mathbf{R}^{n}\right) \cap L^{\infty}\left(\mathbf{R}^{n}\right)$. Therefore, by Lemma 2.3, $D^{\beta} \partial_{x_{1}} v \in L^{\frac{2 \delta}{|\beta|}}\left(\mathbf{R}^{n}\right)$ for $1 \leq|\beta| \leq s$. It follows from Minkowski's inequality and (2.23) that $\left(D^{\beta} v\right)\left(\varphi\left(x^{\prime}\right), x^{\prime}\right) \in L^{\frac{2 s}{|\beta|}}\left(\mathbf{R}^{n-1}\right)$, with norm depending only on $s, n$, the size of the supports, $\left\|v\left(x_{1}, x^{\prime}\right)\right\|_{H^{s+1}}$ and $\|\varphi\|_{L^{\infty}}$.

We notice that $D \varphi \in H^{s}\left(\mathbf{R}^{n-1}\right) \cap L^{\infty}\left(\mathbf{R}^{n-1}\right)$; therefore $D^{\beta} \varphi \in L^{\frac{2 s}{|\beta|-1}}\left(\mathbf{R}^{n-1}\right)$ for $1 \leq|\beta| \leq s$, by Lemma 2.3. The chain rule and the Leibniz formula imply that, for $1 \leq|\gamma| \leq s, D^{\gamma}\left(v\left(\varphi\left(x^{\prime}\right), x^{\prime}\right)\right)$ may be written as a sum of terms of the form

$$
\left(D^{\beta_{0}} D^{m} v\right)\left(\varphi\left(x^{\prime}\right), x^{\prime}\right)\left(D^{\beta_{1}} \varphi\left(x^{\prime}\right)\right) \cdots\left(D^{\beta_{m}} \varphi\left(x^{\prime}\right)\right) \text {, }
$$


where $D^{m}$ stands for a derivative of order $m$ with respect to $x_{1}$ with $0 \leq$ $m \leq s$ and $\beta_{0}+\beta_{1}+\cdots+\beta_{m}=\gamma, 1 \leq\left|\beta_{k}\right| \leq s$ for $k=0,1, \cdots, m$. If $m=$ 0 , then $\gamma=\beta_{0}$, and so $\left(D^{\beta_{0}} v\right)\left(\varphi\left(x^{\prime}\right), x^{\prime}\right) \in L^{\frac{2 s}{\beta_{0} \mid}}\left(\mathbf{R}^{n-1}\right) \subset L^{2}\left(\mathbf{R}^{n-1}\right)$ with $\left\|\left(D^{\beta_{0}} v\right)\left(\varphi\left(x^{\prime}, x^{\prime}\right)\right)\right\|_{L^{2}} \leq C$, where $C$ depends only on the size of supports, $s$, $n,\left\|v\left(x_{1}, x^{\prime}\right)\right\|_{H^{s+1}}$ and $\|\varphi\|_{L^{\infty}}$. Therefore, we may assume that $m \geq 1$. The preceding estimates and Hölder's inequality imply that the use of the chain rule was justified, and

$$
\left(D^{\beta_{0}} D^{m} v\right)\left(\varphi\left(x^{\prime}\right), x^{\prime}\right)\left(D^{\beta_{1}} \varphi\left(x^{\prime}\right)\right) \cdots\left(D^{\beta_{m}} \varphi\left(x^{\prime}\right)\right) \in L^{2}\left(\mathbf{R}^{n-1}\right)
$$

since

$$
\frac{\left|\beta_{0}\right|+m}{2 s}+\frac{\left(\left|\beta_{1}\right|-1\right)+\cdots+\left(\left|\beta_{m}\right|-1\right)}{2 s} \leq \frac{|\gamma|}{2 s} \leq \frac{1}{2} .
$$

Therefore $D^{\gamma}\left(v\left(\varphi\left(x^{\prime}\right), x^{\prime}\right)\right) \in L^{2}\left(\mathbf{R}^{n-1}\right)$ for $1 \leq|\gamma| \leq s$. Moreover, by Lemma 2.3, $\left\|D^{\gamma}\left(v\left(\varphi\left(x^{\prime}\right), x^{\prime}\right)\right)\right\|_{L^{2}}$ is bounded up to an appropriate constant by

$$
\left(\|D \varphi\|_{H^{s}}\right)^{\frac{\left|\beta_{1}\right|-1}{s}} \cdots\left(\|D \varphi\|_{H^{s}}\right)^{\frac{\left|\beta_{m}\right|-1}{s}} .
$$

Since $\left(\left|\beta_{1}\right|-1\right)+\cdots+\left(\left|\beta_{m}\right|-1\right) \leq s-1$, the required estimate holds.

According to Lemma 2.9, we have the following corollary similar to Corollary 2.8.

Corollary 2.10. Let $v\left(t, x_{1}, x^{\prime}\right) \in C\left(\mathbf{R} ; H_{\mathrm{loc}}^{s+1}\left(\mathbf{R}^{n}\right)\right)$ for $s>\frac{n}{2}$. Suppose that $\varphi(t, x) \in C\left(\mathbf{R} ; H_{\mathrm{loc}}^{s+1}\left(\mathbf{R}^{n-1}\right)\right)$, and that $D \varphi\left(t, x^{\prime}\right) \in C\left(\mathbf{R} ; L_{\mathrm{loc}}^{\infty}\left(\mathbf{R}^{n-1}\right)\right)$. Then $v\left(t, \varphi\left(t, x^{\prime}\right), x^{\prime}\right) \in C\left(\mathbf{R} ; H_{\mathrm{loc}}^{s}\left(\mathbf{R}^{n-1}\right)\right)$.

The defining function for the characteristic hypersurface $x_{1}=\varphi\left(t, x^{\prime}\right)$ associated with the quasilinear equation (1.1) satisfies (2.22). Thus $\varphi_{t}$ may be expressed locally as a smooth function of $t, x^{\prime}, u\left(t, \varphi\left(t, x^{\prime}\right), x^{\prime}\right)$, $D u\left(t, \varphi\left(t, x^{\prime}\right), x^{\prime}\right)$ and $\tilde{D} \varphi\left(t, x^{\prime}\right)$, where $\tilde{D} \varphi$ is the $x^{\prime}$ gradient of $\varphi$. Such a function will be denoted by $f\left(v\left(t, \varphi\left(t, x^{\prime}\right), x^{\prime}\right), \tilde{D} \varphi\left(t, x^{\prime}\right)\right)$, with $v$ representing the vector $\left(t, x^{\prime}, u, D u\right)$. From now on, we use $D$ as total derivative and $\tilde{D}$ as $x^{\prime}$ derivative.

Theorem 2.11. Let $v\left(t, x_{1}, x^{\prime}\right) \in C\left(\mathbf{R} ; H_{\mathrm{loc}}^{s+1}\left(\mathbf{R}^{n}\right)\right)$ for $s>\frac{n}{2}+1$, and assume that $D^{2} \varphi\left(t, x^{\prime}\right) \in L_{\mathrm{loc}}^{\infty}\left(\mathbf{R} \times \mathbf{R}^{n-1}\right)$. Let $f$ be a smooth function of its arguments, and suppose that $\varphi_{t}\left(t, x^{\prime}\right)=f\left(v\left(t, \varphi\left(t, x^{\prime}\right), x^{\prime}\right), \tilde{D} \varphi\left(t, x^{\prime}\right)\right)$. If $\varphi\left(0, x^{\prime}\right) \in$ $H_{\mathrm{loc}}^{s}\left(\mathbf{R}^{n-1}\right)$, then $\varphi\left(t, x^{\prime}\right) \in C\left(\mathbf{R} ; H_{\mathrm{loc}}^{s}\left(\mathbf{R}^{n-1}\right)\right)$.

Proof. It can be assumed that the functions in question all have compact support in $x^{\prime}$. Let $\varphi^{(s)}$ denote the vector of all $x^{\prime}$ derivatives of $\varphi$ up to order $s$. Under the assumption that $\varphi$ is smooth, we will establish an $a$ priori estimate on the energy $E(t)=\left(\int\left|\varphi^{(s)}\left(t, x^{\prime}\right)\right|^{2} d x^{\prime}\right)^{\frac{1}{2}}$. Standard arguments then allow the smoothness assumption to be dropped.

The chain rule and the Leibniz formula imply that there are smooth functions $f, F$ and $f_{\alpha}$ for $\left|\alpha_{1}\right|+\cdots+\left|\alpha_{k}\right|+\left|\alpha_{k+1}\right|+\cdots+\left|\alpha_{m}\right| \leq s, k \geq 1$, which are then evaluated at $\left(v\left(t, \varphi\left(t, x^{\prime}\right), x^{\prime}\right), \tilde{D} \varphi\left(t, x^{\prime}\right)\right)$, such that

$$
\begin{aligned}
\partial_{t} \varphi^{(s)}= & F \tilde{D} \varphi^{(s)}\left(t, x^{\prime}\right)+f+\sum_{\alpha} f_{\alpha} \tilde{D}^{\alpha_{1}}\left(v\left(t, \varphi, x^{\prime}\right)\right) \\
& \cdots \tilde{D}^{\alpha_{k}}\left(v\left(t, \varphi, x^{\prime}\right)\right) \tilde{D}^{\alpha_{k+1}}(\tilde{D} \varphi) \cdots \tilde{D}^{\alpha_{m}}(\tilde{D} \varphi) .
\end{aligned}
$$


The energy satisfies $E(t) \partial_{t} E(t)=\int \varphi^{(s)}\left(t, x^{\prime}\right) \partial_{t} \varphi^{(s)}\left(t, x^{\prime}\right) d x^{\prime}$, and by integration by parts,

$$
\begin{aligned}
\int \varphi^{(s)} F(v, \tilde{D} \varphi)\left(\tilde{D} \varphi^{(s)}\right) d x^{\prime} & =\frac{1}{2} \int F(v, \tilde{D} \varphi) \tilde{D}\left(\left(\varphi^{(s)}\right)^{2}\right) d x^{\prime} \\
& =-\frac{1}{2} \int \tilde{D} F(v, \tilde{D} \varphi)\left(\varphi^{(s)}\right)^{2} d x^{\prime} .
\end{aligned}
$$

We note, by the chain rule, that $\tilde{D} F(v, \tilde{D} \varphi)=\sum_{\sigma} F_{\sigma}(\tilde{D} v)+G_{\sigma}\left(\tilde{D}^{2} \varphi\right)$, where $F_{\sigma}$ and $G_{\sigma}$ are smooth functions of their arguments $v, \tilde{D} \varphi$. Since $v\left(t, x_{1}, x^{\prime}\right)$ $\in C\left(\mathbf{R} ; H^{s+1}\left(\mathbf{R}^{n}\right)\right)$ and $s>\frac{n}{2}+1$, the Sobolev imbedding theorem and Corollary 2.8 imply that $v\left(t, \varphi, x^{\prime}\right) \in L_{\mathrm{loc}}^{\infty}\left(\mathbf{R} \times \mathbf{R}^{n}\right), D\left(v\left(t, \varphi, x^{\prime}\right)\right) \in$ $L_{\mathrm{loc}}^{\infty}\left(\mathbf{R} \times \mathbf{R}^{n}\right)$, and since $D^{2} \varphi\left(t, x^{\prime}\right) \in L_{\mathrm{loc}}^{\infty}\left(\mathbf{R} \times \mathbf{R}^{n-1}\right), \tilde{D}\left(F\left(v\left(t, \varphi, x^{\prime}\right)\right.\right.$, $\left.\left.\tilde{D} \varphi\left(t, x^{\prime}\right)\right)\right) \in L_{\mathrm{loc}}^{\infty}\left(\mathbf{R} \times \mathbf{R}^{n-1}\right), f\left(v\left(t, \varphi, x^{\prime}\right), \tilde{D} \varphi\left(t, x^{\prime}\right)\right) \in L_{\mathrm{loc}}^{\infty}\left(\mathbf{R} \times \mathbf{R}^{n-1}\right)$ and $f_{\alpha}\left(v\left(t, \varphi, x^{\prime}\right), \tilde{D} \varphi\left(t, x^{\prime}\right)\right) \in L_{\mathrm{loc}}^{\infty}\left(\mathbf{R} \times \mathbf{R}^{n-1}\right)$.

By Lemma 2.7 and Corollary $2.8, v\left(t, \varphi\left(t, x^{\prime}\right), x^{\prime}\right) \in C\left(\mathbf{R} ; H^{s}\left(\mathbf{R}^{n-1}\right)\right)$ with $\left\|v\left(t, \varphi\left(t, x^{\prime}\right), x^{\prime}\right)\right\|_{H^{s}} \leq C(t) E(t)$. Therefore

$$
\tilde{D}\left(v\left(t, \varphi\left(t, x^{\prime}\right), x^{\prime}\right)\right) \in C\left(\mathbf{R} ; H^{s-1}\left(\mathbf{R}^{n-1}\right)\right) \cap L_{\mathrm{loc}}^{\infty}\left(\mathbf{R} \times \mathbf{R}^{n-1}\right)
$$

with similar bounds on the $H^{s-1}\left(\mathbf{R}^{n-1}\right)$ norm. Hence from Lemma 2.3,

$$
\tilde{D}^{\alpha_{j}}\left(v\left(t, \varphi\left(t, x^{\prime}\right), x^{\prime}\right)\right) \in L^{2 p_{j}}\left(\mathbf{R}^{n-1}\right), \quad \text { where } p_{j}=\frac{s-1}{\left|\alpha_{j}\right|-1}
$$

and

$$
\left\|\tilde{D}^{\alpha_{j}}\left(v\left(t, \varphi\left(t, x^{\prime}\right), x^{\prime}\right)\right)\right\|_{L^{2 p_{j}}} \leq C\|\tilde{D} v\|_{L^{\infty}}^{1-\frac{1}{p_{j}}}\|\tilde{D} v\|_{H^{s-1}}^{\frac{1}{p_{j}}} \leq C(t)(E(t))^{\frac{1}{p_{j}}}
$$
for $1 \leq\left|\alpha_{j}\right| \leq s, j=1, \cdots, k$. Similarly,

$$
(D \varphi) \in C\left(\mathbf{R} ; H^{s-1}\left(\mathbf{R}^{n-1}\right)\right) \cap L_{\text {loc }}^{\infty}\left(\mathbf{R} \times \mathbf{R}^{n-1}\right),
$$

with $\|D \varphi\|_{H^{s-1}} \leq E(t)$ and $\|D \varphi\|_{L^{\infty}} \leq C(t)$. Thus, again from Lemma 2.3,

$$
\tilde{D}^{\alpha_{j}}\left(\tilde{D} \varphi\left(t, x^{\prime}\right)\right) \in L^{2 q_{j}}\left(\mathbf{R}^{n-1}\right), \quad \text { where } q_{j}=\frac{s-1}{\left|\alpha_{j}\right|},
$$

and

$$
\left\|\tilde{D}^{\alpha_{j}}(\tilde{D} \varphi)\right\|_{L^{2 q_{j}}} \leq C\|D \varphi\|_{L^{\infty}}^{1-\frac{1}{q_{j}}}\|D \varphi\|_{H^{s-1}}^{\frac{1}{q_{j}}} \leq C(t)(E(t))^{\frac{1}{q_{j}}},
$$

for $0 \leq\left|\alpha_{j}\right| \leq s-1, j=k+1, \cdots, m$. Therefore, by Hölder's inequality,

$$
\begin{aligned}
& \tilde{D}^{\alpha_{1}}\left(v\left(t, \varphi\left(t, x^{\prime}\right), x^{\prime}\right)\right) \cdots \tilde{D}^{\alpha_{k}}\left(v\left(t, \varphi\left(t, x^{\prime}\right), x^{\prime}\right)\right) \tilde{D}^{\alpha_{k+1}}(\tilde{D} \varphi) \cdots \tilde{D}^{\alpha_{m}}(\tilde{D} \varphi) \\
& \in C\left(\mathbf{R} ; L^{2}\left(\mathbf{R}^{n-1}\right)\right) \text {, }
\end{aligned}
$$

since $\left(\left(\left|\alpha_{1}\right|-1\right)+\cdots+\left(\left|\alpha_{k}\right|-1\right)+\left|\alpha_{k+1}\right|+\cdots+\left|\alpha_{m}\right|\right) / 2(s-1) \leq(s-k) / 2(s-1) \leq$ $1 / 2$. From (2.24) and (2.25),

$$
\begin{gathered}
\left\|\left(\tilde{D}^{\alpha_{1}} v\right) \cdots\left(\tilde{D}^{\alpha_{k}} v\right) \tilde{D}^{\alpha_{k+1}}(\tilde{D} \varphi) \cdots \tilde{D}^{\alpha_{m}}(\tilde{D} \varphi)\right\|_{L^{2}} \\
\leq C(t)(E(t))^{\frac{s-k}{s-1}} \leq C(t) E(t) .
\end{gathered}
$$


By Minkowski's inequality, Schwarz's inequality and (2.26),

$$
\begin{gathered}
\left|E(t) \partial_{t} E(t)\right| \leq C\|\tilde{D} F\|_{L^{\infty}} \int\left(\varphi^{(s)}\right)^{2} d x^{\prime} \\
+C\left(\int\left(\varphi^{(s)}\right)^{2} d x^{\prime}\right)^{\frac{1}{2}}\left[\|f\|_{L^{\infty}}+\sum_{\alpha} \| f_{\alpha}\left(\tilde{D}^{\alpha_{1}} v\left(t, \varphi, x^{\prime}\right)\right)\right. \\
\left.\cdots\left(\tilde{D}^{\alpha_{k}} v\left(t, \varphi, x^{\prime}\right)\right) \tilde{D}^{\alpha_{k+1}}(\tilde{D} \varphi) \cdots \tilde{D}^{\alpha_{m}}(\tilde{D} \varphi) \|_{L^{2}}\right] \\
\leq C E(t)\left(\|\tilde{D} F\|_{L^{\infty}} E(t)+\|f\|_{L^{\infty}}+\left\|f_{\alpha}\right\|_{L^{\infty}} C(t) E(t)\right),
\end{gathered}
$$

and so $\left|\partial_{t} E(t)\right| \leq C(t) E(t)+C(t)$. Therefore, by Gronwall's inequality, $E(t)$ is finite for all time since by assumption it is finite at $t=0$.

We will also encounter first order equations like those in Theorem 2.11 which are linear, but with coefficients of finite regularity.

Corollary 2.12. Let $\psi\left(t, x^{\prime}\right)$ satisfy the equation

$$
\psi_{t}=F_{1}\left(t, x^{\prime}\right) \tilde{D} \psi+F_{2}\left(t, x^{\prime}\right) \psi+F_{3}\left(t, x^{\prime}\right)
$$

where

$$
F_{1}\left(t, x^{\prime}\right) \in C\left(\mathbf{R} ; H_{\text {loc }}^{s-1}\left(\mathbf{R}^{n-1}\right)\right)
$$

and

$$
F_{2}\left(t, x^{\prime}\right), F_{3}\left(t, x^{\prime}\right) \in C\left(\mathbf{R} ; H_{\mathrm{loc}}^{s-2}\left(\mathbf{R}^{n-1}\right)\right), \quad s>\frac{n}{2}+2 .
$$

If $\psi\left(0, x^{\prime}\right) \in H_{\mathrm{loc}}^{s-2}\left(\mathbf{R}^{n-1}\right)$, then $\psi\left(t, x^{\prime}\right) \in C\left(\mathbf{R} ; H_{\mathrm{loc}}^{s-2}\left(\mathbf{R}^{n-1}\right)\right)$.

Proof. It can be assumed that the functions in question all have support in $x^{\prime}$. Let $\psi^{(s-2)}$ denote the vector of all $x^{\prime}$ derivatives of $\psi$ up to order $s-2$. As we see in the proof of Theorem 2.11, we will establish a priori estimates on the energy $E(t)=\left(\int\left|\psi^{(s-2)}\left(t, x^{\prime}\right)\right|^{2} d x^{\prime}\right)^{\frac{1}{2}}$.

The chain rule and the Leibniz formula imply that for $\left|\alpha_{1}\right|+\left|\alpha_{2}\right| \leq s-1,1 \leq$ $\left|\alpha_{2}\right| \leq s-2$ and $\left|\beta_{1}\right|+\left|\beta_{2}\right| \leq s-2$,

$$
\begin{aligned}
\partial_{t} \psi^{(s-2)}=F_{1}\left(t, x^{\prime}\right) \tilde{D} \psi^{(s-2)}\left(t, x^{\prime}\right)+\sum_{\alpha}\left(\tilde{D}^{\alpha_{1}} F_{1}\left(t, x^{\prime}\right)\right)\left(\tilde{D}^{\alpha_{2}} \psi\left(t, x^{\prime}\right)\right) \\
+\sum_{\beta}\left(\tilde{D}^{\beta_{1}} F_{2}\left(t, x^{\prime}\right)\right)\left(\tilde{D}^{\beta_{2}} \psi\left(t, x^{\prime}\right)\right)+\tilde{D}^{s-2} F_{3}\left(t, x^{\prime}\right) .
\end{aligned}
$$

The energy satisfies $E(t) \partial_{t} E(t)=\int \psi^{(s-2)}\left(t, x^{\prime}\right) \partial_{t} \psi^{(s-2)}\left(t, x^{\prime}\right) d x^{\prime}$, and by integration by parts,

$$
\int \psi^{(s-2)} F_{1}\left(t, x^{\prime}\right)\left(\tilde{D} \psi^{(s-2)}\right) d x^{\prime}=-\frac{1}{2} \int \tilde{D} F_{1}\left(t, x^{\prime}\right)\left(\psi^{(s-2)}\right)^{2} d x^{\prime} .
$$

Since $s>\frac{n}{2}+2$, the Sobolev imbedding theorem implies that $\tilde{D} F_{1}\left(t, x^{\prime}\right) \in$ $L_{\text {loc }}^{\infty}\left(\mathbf{R} \times \mathbf{R}^{n-1}\right)$.

Since $\tilde{D} F_{1}\left(t, x^{\prime}\right) \in C\left(\mathbf{R} ; H^{s-2}\left(\mathbf{R}^{n-1}\right)\right) \cap L_{\text {loc }}^{\infty}\left(\mathbf{R} \times \mathbf{R}^{n-1}\right)$, by Lemma 2.3,

$$
\tilde{D}^{\alpha_{1}} F_{1}\left(t, x^{\prime}\right) \in L^{2 p_{1}}\left(\mathbf{R}^{n-1}\right), \quad \text { where } \quad p_{1}=\frac{s-2}{\left|\alpha_{1}\right|-1}
$$


and

$$
\left\|\tilde{D}^{\alpha_{1}} F_{1}\left(t, x^{\prime}\right)\right\|_{L^{2 p_{1}}} \leq C\left\|\tilde{D} F_{1}\right\|_{L^{\infty}}^{1-\frac{1}{p_{1}}}\left\|\tilde{D} F_{1}\right\|_{H^{s-2}}^{\frac{1}{p_{1}}} \leq C(t) .
$$

for $1 \leq\left|\alpha_{1}\right| \leq s-1$. Similarly,

$$
\psi \in C\left(\mathbf{R} ; H^{s-2}\left(\mathbf{R}^{n-1}\right)\right) \cap L_{\text {loc }}^{\infty}\left(\mathbf{R} \times \mathbf{R}^{n-1}\right),
$$

with $\|\psi\|_{H^{s-2}} \leq E(t)$ and $\|\psi\|_{L^{\infty}} \leq C(t)$. Thus, again from Lemma 2.3,

$$
\tilde{D}^{\alpha_{2}} \psi\left(t, x^{\prime}\right) \in L^{2 p_{2}}\left(\mathbf{R}^{n-1}\right), \quad \text { where } \quad p_{2}=\frac{s-2}{\left|\alpha_{2}\right|}
$$

and

$$
\left\|\tilde{D}^{\alpha_{2}}(\psi)\right\|_{L^{2 p_{2}}} \leq C\|\psi\|_{L^{\infty}}^{1-\frac{1}{p_{2}}}\|\psi\|_{H^{s-2}}^{\frac{1}{p_{2}}} \leq C(t)(E(t))^{\frac{1}{p_{2}}},
$$

for $1 \leq\left|\alpha_{2}\right| \leq s-2$. Therefore, by Hölder's inequality,

$$
\left(\tilde{D}^{\alpha_{1}} F_{1}\left(t, x^{\prime}\right)\right)\left(\tilde{D}^{\alpha} \psi\right) \in C\left(\mathbf{R} ; L^{2}\left(\mathbf{R}^{n-1}\right)\right),
$$

since $\left(\left(\left|\alpha_{1}\right|-1\right)+\left|\alpha_{2}\right|\right) / 2(s-2) \leq 1 / 2$. For $\left|\alpha_{1}\right|=0,\left\|F_{1}\left(t, x^{\prime}\right) \tilde{D} \psi\left(t, x^{\prime}\right)\right\|_{L^{2}} \leq$ $C\left\|F_{1}\right\|_{L^{\infty}} E(t)$. Therefore, from (2.28) and (2.29),

$$
\left\|\left(\tilde{D}^{\alpha_{1}} F_{1}\left(t, x^{\prime}\right)\right)\left(\tilde{D}^{\alpha_{2}} \psi\left(t, x^{\prime}\right)\right)\right\|_{L^{2}} \leq C(t) E(t) .
$$

Since $F_{2}\left(t, x^{\prime}\right) \in C\left(\mathbf{R} ; H^{s-2}\left(\mathbf{R}^{n-1}\right)\right) \cap L_{\text {loc }}^{\infty}\left(\mathbf{R} \times \mathbf{R}^{n-1}\right)$, from Lemma 2.3,

$$
\tilde{D}^{\beta_{1}}\left(F_{2}\left(t, x^{\prime}\right)\right) \in L^{2 q_{1}}\left(\mathbf{R}^{n-1}\right), \quad \text { where } \quad q_{1}=\frac{s-2}{\left|\beta_{1}\right|}
$$

and

$$
\left\|\tilde{D}^{\beta_{1}}\left(F_{2}\left(t, x^{\prime}\right)\right)\right\|_{L^{2 q_{1}}} \leq C\left\|F_{2}\right\|_{L^{\infty}}^{1-\frac{1}{p_{j}}}\left\|F_{2}\right\|_{H^{s-2}}^{\frac{1}{q_{1}}} \leq C(t),
$$

for $0 \leq\left|\beta_{1}\right| \leq s-2$. Also, again from Lemma 2.3,

$$
\left.\tilde{D}^{\beta_{2}} \psi\left(t, x^{\prime}\right)\right) \in L^{2 q_{2}}\left(\mathbf{R}^{n-1}\right), \quad \text { where } \quad q_{2}=\frac{s-2}{\left|\beta_{2}\right|},
$$

and

$$
\left\|\tilde{D}^{\beta_{2}} \psi\right\|_{L^{2 q_{2}}} \leq C\|\psi\|_{L^{\infty}}^{1-\frac{1}{q_{2}}}\|\psi\|_{H^{s-2}}^{\frac{1}{q_{2}}} \leq C(t)(E(t))^{\frac{1}{q_{2}}},
$$

for $0 \leq\left|\beta_{2}\right| \leq s-2$. Therefore, by Hölder's inequality,

$$
\tilde{D}^{\beta_{1}}\left(F_{2}\left(t, x^{\prime}\right)\right) \tilde{D}^{\beta_{2}} \psi \in C\left(\mathbf{R} ; L^{2}\left(\mathbf{R}^{n-1}\right)\right),
$$

since $\left(\left|\beta_{1}\right|+\left|\beta_{2}\right|\right) / 2(s-2) \leq 1 / 2$. From (2.31) and (2.32),

$$
\left\|\tilde{D}^{\beta_{1}}\left(F_{2}\left(t, x^{\prime}\right)\right) \tilde{D}^{\beta_{2}} \psi\left(t, x^{\prime}\right)\right\|_{L^{2}} \leq C(t) E(t) .
$$

Therefore, by Minkowski's inequality, Schwarz's inequality, (2.31) and (2.33),

$$
\begin{aligned}
\left|E(t) \partial_{t} E(t)\right| \leq C\left\|\tilde{D} F_{1}\right\|_{L^{\infty}} \int\left(\varphi^{(s-2)}\right)^{2} d x^{\prime}+C\left(\int\left(\varphi^{(s-2)}\right)^{2} d x^{\prime}\right)^{\frac{1}{2}} \\
+\left[\sum_{\alpha}\left\|\left(\tilde{D}^{\alpha_{1}} F_{1}\right)\left(\tilde{D}^{\alpha_{2}} \psi\right)\right\|_{L^{2}}+\sum_{\beta}\left\|\left(\tilde{D}^{\beta_{1}} F_{2}\right)\left(\tilde{D}^{\beta_{2}} \psi\right)\right\|_{L^{2}}+\left\|\tilde{D}^{s-2} F_{3}\right\|_{L^{2}}\right] \\
\leq C E(t)\left(\left\|\tilde{D} F_{1}\right\|_{L^{\infty}} E(t)+C(t) E(t)+C(t)\right),
\end{aligned}
$$


and so $\left|\partial_{t} E(t)\right| \leq C(t) E(t)+C(t)$. Therefore, by Gronwall's inequality, $E(t)$ is finite for all time since by assumption it is finite at $t=0$.

\section{CONORMAL SOLUTIONS FOR QUASILINEAR WAVE EQUATIONS}

In this section, we will consider the conormal regularity of solutions of equation (1.1) with respect to a single characteristic hypersurface and a pair of characteristic hypersurfaces which intersect transversally.

\subsection{Conormal regularity with respect to a single characteristic hypersurface.}

Definition 3.1. Let $\Sigma$ be a $C^{2}$ characteristic hypersurface for $P_{2}$ given in (1.1). $w(t, x) \in C\left(\mathbf{R} ; H_{\mathrm{loc}}^{\tau}\left(\mathbf{R}^{n}\right)\right) \cap C^{1}\left(\mathbf{R} ; H_{\mathrm{loc}}^{\tau-1}\left(\mathbf{R}^{n}\right)\right)$ is said to be conormal with respect to $\Sigma$ if $M_{1} \cdots M_{j} w \in C\left(\mathbf{R} ; H_{\mathrm{loc}}^{\tau}\left(\mathbf{R}^{n}\right)\right) \cap C^{1}\left(\mathbf{R} ; H_{\mathrm{loc}}^{\tau-1}\left(\mathbf{R}^{n}\right)\right)$ for all $C\left(\mathbf{R} ; H_{\text {loc }}^{\tau}\left(\mathbf{R}^{n}\right)\right) \cap C^{1}\left(\mathbf{R} ; H_{\text {loc }}^{\tau-1}\left(\mathbf{R}^{n}\right)\right)$ vector fields $M_{1}, \cdots, M_{j}$ which are tangent to $\Sigma$. It will be denoted by $w \in N^{\tau, \infty}(\Sigma)$. If this property holds for all $j \leq k$, then $u$ is said to be conormal of degree $k$ with respect to $\Sigma$, denoted by $w \in N^{\tau, k}(\Sigma)$.

Since surfaces $\{t=$ constant $\}$ are space-like, it follows that we can assume without loss of generality that a characteristic hypersurface $\Sigma$ for $P_{2}$ is given locally by $\left\{x_{1}=\varphi\left(t, x^{\prime}\right)\right\}$ with nonvanishing gradient of the function $\varphi\left(t, x^{\prime}\right)$, and we suppose that $u \in C\left(\mathbf{R} ; H_{\mathrm{loc}}^{s}\left(\mathbf{R}^{n}\right)\right) \cap C^{!}\left(\mathbf{R} ; H_{\mathrm{loc}}^{s-1}\left(\mathbf{R}^{n}\right)\right), s>\frac{n}{2}+4$. Then $\varphi$ satisfies (2.22), where coefficients are evaluated at $x_{1}=\varphi\left(t, x^{\prime}\right)$ and $\varphi \in$ $C\left(\mathbf{R} ; H_{\mathrm{loc}}^{s-2}\left(\mathbf{R}^{n-1}\right)\right) \cap C^{1}\left(\mathbf{R} ; H_{\mathrm{loc}}^{s-3}\left(\mathbf{R}^{n-1}\right)\right) \subset C^{2}\left(\mathbf{R} \times \mathbf{R}^{n-1}\right)$, by Theorem 2.11 .

Consider a change of variables:

$$
y_{0}=t, \quad y_{1}=x_{1}-\varphi\left(t, x^{\prime}\right), \quad y_{2}=x_{2}, \cdots, \quad y_{n}=x_{n},
$$

with inverse transform:

$$
t=y_{0}, \quad x_{1}=y_{1}+\varphi\left(y_{0}, y^{\prime}\right), \quad x_{2}=y_{2}, \cdots, \quad x_{n}=y_{n},
$$

where $y^{\prime}=\left(y_{2}, \cdots, y_{n}\right) \in \mathbf{R}^{n-1}$. Under the change of variables (3.1), $\Sigma$ becomes locally $S=y_{1}=0$ in the $y$-coordinates. We notice that $\frac{\partial}{\partial y_{0}}, y_{1} \frac{\partial}{\partial y_{1}}, \frac{\partial}{\partial y_{2}}$, $\cdots, \frac{\partial}{\partial y_{n}}$ generate the tangential vector fields to $S$. Therefore, by the chain rule, the $\left.C\left(\mathbf{R} ; H_{\text {loc }}^{s-2} \mathbf{R}^{n}\right)\right) \cap C^{1}\left(\mathbf{R} ; H_{\text {loc }}^{s-3}\left(\mathbf{R}^{n}\right)\right)$ vector fields tangent to $\Sigma$ are spanned by

$$
\begin{aligned}
& M_{0}=\left(x_{1}-\varphi\left(t, x^{\prime}\right)\right) \partial_{x_{1}}, M_{1}=\partial_{t}+\varphi_{t}\left(t, x^{\prime}\right) \partial_{x_{1}} \\
& M_{j}=\partial_{x_{j}}+\varphi_{x_{j}}\left(t, x^{\prime}\right) \partial_{x_{1}}, \quad 2 \leq j \leq n
\end{aligned}
$$

The idea of the proof of conormal regularity is to use commutator arguments and (1.1) to inductively establish regularity for $u$ with respect to differentiation by the vector fields given by (3.2), for coefficients of a given regularity. The improved regularity of $u$, coupled with (2.22), then yields improved regularity of $\varphi$, and hence of the coefficients of the vector fields, allowing the inductive argument to be continued. 
We note the following: For $2 \leq i, j \leq n$,

$$
\begin{aligned}
\partial_{t} \partial_{x_{1}} & =\partial_{x_{1}}\left(\partial_{t}+\varphi_{t} \partial_{x_{1}}-\varphi_{t} \partial_{x_{1}}\right)=\partial_{x_{1}} M_{1}-\varphi_{t} \partial_{x_{1}}^{2} \\
\partial_{t} \partial_{x_{j}} & =\partial_{t}\left(\partial_{x_{j}}+\varphi_{x_{j}} \partial_{x_{1}}-\varphi_{x_{j}} \partial_{x_{1}}\right)=\partial_{t} M_{j}-\varphi_{t x_{j}} \partial_{x_{1}}-\varphi_{x_{j}} \partial_{t} \partial_{x_{1}} \\
\partial_{x_{1}} \partial_{x_{j}} & =\partial_{x_{1}}\left(\partial_{x_{j}}+\varphi_{x_{j}} \partial_{x_{1}}-\varphi_{x_{j}} \partial_{x_{1}}\right)=\partial_{x_{1}} M_{j}-\varphi_{x_{j}} \partial_{x_{1}}^{2} \\
\partial_{x_{i}} \partial_{x_{j}} & =\partial_{x_{i}}\left(\partial_{x_{j}}+\varphi_{x_{j}} \partial_{x_{1}}-\varphi_{x_{j}} \partial_{x_{1}}\right)=\partial_{x_{i}} M_{j}-\varphi_{x_{i} x_{j}} \partial_{x_{1}}-\varphi_{x_{j}} \partial_{x_{i}} \partial_{x_{1}} .
\end{aligned}
$$

Let

$$
F\left(t, x_{1}, x^{\prime}\right)=\left(\varphi_{t}\right)^{2}-a_{11}+2\left(a_{01}-\sum_{j=2}^{n} a_{0 j}\right) \varphi_{t}+2 \sum_{j=2}^{n} a_{1 j} \varphi_{x_{j}}-\sum_{i, j=2}^{n} a_{i j} \varphi_{x_{i}} \varphi_{x_{j}},
$$

where

$$
a_{i j}=a_{i j}\left(t, x_{1}, x^{\prime}, u\left(t, x_{1}, x^{\prime}\right), D u\left(t, x_{1}, x^{\prime}\right)\right) .
$$

Since $F\left(t, \varphi\left(t, x^{\prime}\right), x^{\prime}\right) \equiv 0$, Taylor's theorem and (2.22) imply that

$$
\begin{aligned}
F\left(t, x_{1}, x^{\prime}\right) & =\left(x_{1}-\varphi\right)\left(\int_{0}^{1} F_{1}\left(t, s x_{1}+(1-s) \varphi, x^{\prime}\right) d s\right) \\
& \equiv\left(x_{1}-\varphi\right) \tilde{F}\left(t, x_{1}, x^{\prime}\right),
\end{aligned}
$$

where $F_{1}\left(t, x_{1}, x^{\prime}\right)=\frac{\partial F}{\partial x_{1}}\left(t, x_{1}, x^{\prime}\right)$. We note that by the chain rule, $\tilde{F}$ may be considered as an integral of a smooth function of $t, x^{\prime}, u, D u, D^{2} u, \varphi$ and $D \varphi$. Therefore,

$$
\begin{gathered}
a_{11}=\left(\varphi_{t}\right)^{2}+2\left(a_{01}-\sum_{j=2}^{n} a_{0 j} \varphi_{x_{j}}\right) \varphi_{t}+2 \sum_{j=2}^{n} a_{1 j} \varphi_{x_{j}}-\sum_{i, j=2}^{n} a_{i j} \varphi_{x_{i}} \varphi_{x_{j}} \\
+\left(x_{1}-\varphi\left(t, x^{\prime}\right)\right) \tilde{F}\left(t, x_{1}, x^{\prime}\right) .
\end{gathered}
$$

Substituting $a_{11}$ into (1.2) and using the note above,

$$
\begin{aligned}
P_{2}(t, x, u, D u, D)= & \partial_{t}^{2}-\left(\varphi_{t}\right)^{2} \partial_{x_{1}}^{2}-2 a_{01}\left(\partial_{t} \partial_{x_{1}}+\varphi_{t} \partial_{x_{1}}^{2}\right) \\
& -2 \sum_{j=2}^{n} a_{0 j}\left(\partial_{t} \partial_{x_{j}}-\varphi_{x_{j}} \varphi_{t} \partial_{x_{1}}^{2}\right)-2 \sum_{j=2}^{n} a_{1 j}\left(\partial_{x_{1}} \partial_{x_{j}}+\varphi_{x_{j}} \partial_{x_{1}}^{2}\right) \\
& -\sum_{i, j=2}^{n} a_{i j}\left(\partial_{x_{i}} \partial_{x_{j}}-\varphi_{x_{i}} \varphi_{x_{j}} \partial_{x_{1}}^{2}\right)-\tilde{F}\left(t, x_{1}, x^{\prime}\right)\left(x_{1}-\varphi\right) \partial_{x_{1}}^{2} \\
= & \left(\partial_{t}-\varphi_{t} \partial_{x_{1}}\right) M_{1}-\varphi_{t t} \partial_{x_{1}}-2 a_{01} M_{1} \\
& -2 \sum_{j=2}^{n} a_{0 j}\left(\partial_{t} M_{j}-\varphi_{x_{j}} \partial_{x_{1}} M_{1}-\varphi_{t x_{j}} \partial_{x_{1}}\right)-2 \sum_{j=2}^{n} a_{1 j} \partial_{x_{1}} M_{j} \\
& -\sum_{i, j=2}^{n} a_{i j}\left(\partial_{x_{j}} M_{j}-\varphi_{x_{j}} \partial_{x_{1}} M_{j}-\varphi_{x_{i} x_{j}} \partial_{x_{1}}\right) \\
& -\tilde{F}\left(t, x_{1}, x^{\prime}\right)\left(\partial_{x_{1}} M_{0}-\partial_{x_{1}}\right) .
\end{aligned}
$$

Therefore,

$$
P_{2}(t, x, u, D u, D)=\sum_{i=0}^{n} a_{i}(D) M_{i}+b(D),
$$


where $a_{j}(D)$ is a first order operator and the coefficients of the operator are smooth functions and integrals of smooth functions of $u, D u, D^{2} u, \varphi$ and $D \varphi$, which will be written symbolically as $a_{j}\left(D^{2} u, D \varphi, D\right)$. Similarly, $b(D)$ is a first order operator and $b_{j}(D)=b\left(D^{2} u, D^{2} \varphi, D\right)$.

Since $\left[M_{i}, M_{j}\right]=0,\left[a_{i}(D) M_{i}, M_{j}\right]=c_{i j}(D) M_{i}$ and $\left[b(D), M_{j}\right]=d_{j}(D)$, for $0 \leq i, j \leq n$, where

$$
c_{i j}(D)=c_{i j}\left(D^{3} u, D^{2} \varphi, D\right) \text { and } d_{j}(D)=d_{j}\left(D^{3} u, D^{3} \varphi, D\right)
$$

are first order operators, we have the following lemma.

Lemma 3.1. Let $P_{2}$ be the second order quasilinear differential operator given in (1.2). Let $\Sigma$ be a characteristic hypersurface for $P_{2}$ given locally by $\left\{x_{1}=\right.$ $\left.\varphi\left(t, x^{\prime}\right)\right\}$ with $D \varphi \neq 0$ and $\varphi \in C^{3}\left(\mathbf{R} \times \mathbf{R}^{n}\right)$. Let $u \in C\left(\mathbf{R} ; H_{\mathrm{loc}}^{s}\left(\mathbf{R}^{n}\right)\right) \cap$ $C^{1}\left(\mathbf{R} ; H_{\mathrm{loc}}^{s-1}\left(\mathbf{R}^{n}\right)\right), s>\frac{n}{2}+4$. Then the $C\left(\mathbf{R} ; H_{\mathrm{loc}}^{s-2}\left(\mathbf{R}^{n}\right)\right) \cap C^{1}\left(\mathbf{R} ; H_{\mathrm{loc}}^{s-3}\left(\mathbf{R}^{n}\right)\right)$ vector fields tangent to $\Sigma$ are spanned by $M_{0}, M_{1}, \cdots, M_{n}$ and

$$
\left[P_{2}, M_{j}\right]=\sum_{i=0}^{n}\left(c_{i j}(D) M_{j}+d_{j}(D)\right), \quad \text { for } j=0,1, \cdots, n,
$$

where $c_{i j}(D)=c_{i j}\left(D^{3} u, D^{2} \varphi, D\right)$ and $d_{j}(D)=d_{j}\left(D^{3} u, D^{3} \varphi, D\right)$ are first order operators.

Now, we state and prove the conormal regularity of a solution of equation (1.1) with respect to a single characteristic hypersurface.

Theorem 3.2. Let $P_{2}$ be as above and let $\Sigma$ be a characteristic hypersurface for $P_{2}$, defined by a function with nonvanishing gradient. Suppose that $u \in$ $C\left(\mathbf{R} ; H_{\mathrm{loc}}^{s}\left(\mathbf{R}^{n}\right)\right) \cap C^{1}\left(\mathbf{R} ; H_{\mathrm{loc}}^{s-1}\left(\mathbf{R}^{n}\right)\right), s>\frac{n}{2}+4$, and $u$ satisfies the equation

$$
P_{2}(t, x, u, D u, D) u=f(t, x, u, D u),
$$

where $f$ is smooth. If $\Sigma$ is smooth in $\{t<0\}$ and $u \in N^{s, \infty}(\Sigma \cap\{t<0\})$, then $\Sigma$ is smooth for all time and $u \in N^{s, \infty}(\Sigma)$.

Proof. By finite propagation speed and an analysis local in time, it may assumed that $u$ has compact support in $x$ for each $t$, and that $\Sigma$ is given by $\left\{x_{1}=\right.$ $\left.\varphi\left(t, x^{\prime}\right)\right\}$. Then $\varphi$ satisfies (2.22), which is a nonlinear equation of first order with $C^{2}\left(\mathbf{R}^{n}\right)$ coefficients, by Schauder's lemma, strict hyperbolicity of $P_{2}$ and $\Sigma$ being a characteristic hypersurface of $P_{2}$, because $D u \in C\left(\mathbf{R} ; H^{s-1}\left(\mathbf{R}^{n}\right)\right) \cap$ $C^{1}\left(\mathbf{R} ; H^{s-2}\left(\mathbf{R}^{n}\right)\right)$ with $s-1>\frac{n}{2}+3$. Therefore, $\varphi \in C^{3}\left(\mathbf{R} \times \mathbf{R}^{n-1}\right)$. Let $v=(u, D u)$, and set $s_{0}=s-2$. Then, from (2.22), the hypotheses of Theorem 2.11 are satisfied for $s_{0}$, and therefore

$$
\varphi\left(t, x^{\prime}\right) \in C\left(\mathbf{R} ; H^{s_{0}}\left(\mathbf{R}^{n-1}\right)\right) .
$$

Since the regularity of the coefficients of the vector fields $M_{j}$ is a priori lower than that of $u$, it is necessary to differentiate equation (3.5) in order to use the commutator argument on an appropriate derivative of $u$. Let $U$ stand for the vector of all derivatives of $u$ up to fourth order. From (3.5) and the assumption on $u, U \in C\left(\mathbf{R} ; H^{s-4}\left(\mathbf{R}^{n}\right)\right) \cap C^{1}\left(\mathbf{R} ; H^{s-5}\left(\mathbf{R}^{n}\right)\right)$. Then, by the fact that $\left[P_{2}, D\right]=a\left(D^{2}\right)$, where $a\left(D^{2}\right)=a\left(D^{2} u, D^{2}\right)$ is a second order operator,

$$
P_{2}(t, x, u, D u, D) U=g(D U),
$$


where $g$ is a smooth function. The right-hand side is meaningful even if $s-5 \leq$ $\frac{n}{2}$, because $g$ is linear in the highest order components of $D U$, and the product of a function in $H^{s-4}\left(\mathbf{R}^{n}\right)$ with a function in the $H^{s-5}\left(\mathbf{R}^{n}\right)$ is in $H^{s-5}\left(\mathbf{R}^{n}\right)$ for $s-4>\frac{n}{2}$. Let $M$ stand for the vector operator $\left(1, M_{0}, \cdots, M_{n}\right)$. We note that $\left[M_{i}, D\right]=h_{i}(D)$, for $i=0,1, \cdots, n$, where $h_{i}(D)=h_{i}\left(D^{2} \varphi, D\right)$ is a first order operator. From(3.7) and Lemma 3.1,

$$
\begin{aligned}
P_{2}(t, x, u, D u, D) M U & =\left[P_{2}, M\right] U+M(g(D U)) \\
& =c(D) M U+d(D) U+(M D U) g^{\prime}(D U) \\
& =P_{1}(D) M U+G(D U),
\end{aligned}
$$

where $G(D U)=G\left(D U, D^{3} \varphi\right)$ and $c(D)=c\left(D^{3} u, D^{2} \varphi, D\right), d(D)=$ $d\left(D^{3} u, D^{3} \varphi, D\right)$ and $P_{1}(D)=P_{1}\left(D U, D^{2} \varphi, D\right)$ are first order operators.

The coefficients of $P_{2}$ are in $C\left(\mathbf{R} ; H^{s-1}\left(\mathbf{R}^{n}\right)\right) \cap C^{1}\left(\mathbf{R} ; H^{s-2}\left(\mathbf{R}^{n}\right)\right)$, while by (3.6) and the regularity of $U, G(D U)$ and the coefficients of $P_{1}\left(D U, D^{2} \varphi, D\right)$ are in the space $C\left(\mathbf{R} ; H^{s-5}\left(\mathbf{R}^{n}\right)\right)$. It then follows from (3.8) and Corollary 2.6 that

$$
M U \in C\left(\mathbf{R} ; H^{s-4}\left(\mathbf{R}^{n}\right)\right) \cap C^{1}\left(\mathbf{R} ; H^{s-5}\left(\mathbf{R}^{n}\right)\right) .
$$

In order to use the improved regularity of $U$, it is convenient to differentiate (2.22) two times. It follows that there are smooth functions $f_{j}$ such that

$$
\begin{gathered}
\left(D^{2} \varphi\right)_{t}=f_{1}\left(D u\left(t, \varphi, x^{\prime}\right), \tilde{D} \varphi\right) \tilde{D}\left(D^{2} \varphi\right)+f_{2}\left(D^{2} u\left(t, \varphi, x^{\prime}\right), D^{2} \varphi\right) \\
+f_{3}\left(D^{3} u\left(t, \varphi, x^{\prime}\right), D \varphi\right) .
\end{gathered}
$$

Since $\left(M_{1} v\right)\left(t, \varphi, x^{\prime}\right)=\partial_{t}\left(v\left(t, \varphi, x^{\prime}\right)\right)$ and $\left(M_{j} v\right)\left(t, \varphi, x^{\prime}\right)=\partial_{x_{j}}\left(v\left(t, \varphi, x^{\prime}\right)\right)$ for $2 \leq j \leq n$, it follows from applying $M$ to (3.10) that there are smooth functions $F_{j}$ such that

$$
\begin{aligned}
\left(D^{3} \varphi\right)_{t}= & F_{1}\left(D u\left(t, \varphi, x^{\prime}\right), \tilde{D} \varphi\right) \tilde{D}\left(D^{3} \varphi\right)+F_{2}\left(D^{2} u\left(t, \varphi, x^{\prime}\right), D^{2} \varphi\right)\left(D^{3} \varphi\right) \\
& +F_{3}\left(M D^{3} u\left(t, \varphi, x^{\prime}\right), D^{2} \varphi\right) .
\end{aligned}
$$

Since $D M D^{3} u=M D^{4} u+[D, M] D^{3} u \in C\left(\mathbf{R} ; H^{s-4}\left(\mathbf{R}^{n}\right)\right)$, we have $M D^{3} u \in$ $C\left(\mathbf{R} ; H^{s-3}\left(\mathbf{R}^{n}\right)\right)$. Corollary 2.12 implies that

$$
M D^{3} u\left(t, \varphi, x^{\prime}\right) \in C\left(\mathbf{R} ; H^{s-4}\left(\mathbf{R}^{n-1}\right)\right) \text {. }
$$

Therefore, by Corollary 2.12 for $\psi=D^{3} \varphi, D^{3} \varphi \in C\left(\mathbf{R} ; H^{s_{0}-2}\left(\mathbf{R}^{n-1}\right)\right)$, that is, $\varphi \in C\left(\mathbf{R} ; H^{s_{0}+1}\left(\mathbf{R}^{n-1}\right)\right) \cap C^{1}\left(\mathbf{R} ; H^{s_{0}}\left(\mathbf{R}^{n-1}\right)\right)$.

Suppose inductively that (3.6) is improved to $\varphi \in C\left(\mathbf{R} ; H^{s_{0}+k}\left(\mathbf{R}^{n-1}\right)\right)$ and (3.9) is improved to $M^{k+1} U \in C\left(\mathbf{R} ; H^{s-4}\left(\mathbf{R}^{n}\right)\right) \cap C^{1}\left(\mathbf{R} ; H^{s-5}\left(\mathbf{R}^{n}\right)\right)$. From the analogue of (3.11) for $D^{k+3} \varphi$ and Corollary 2.12 for $\psi=D^{k+3} \varphi$, it follows that

$$
\varphi \in C\left(\mathbf{R} ; H^{s_{0}+k+1}\left(\mathbf{R}^{n-1}\right)\right) \cap \cdots \cap C^{k+1}\left(\mathbf{R} ; H^{s_{0}}\left(\mathbf{R}^{n-1}\right)\right) .
$$

Then, an equation similar to (3.8) holds for $M^{k+2} U$ with coefficients depending smoothly on $D M^{k+1} U$ and $D^{k+3} \varphi$, that is,

$$
P_{2}(t, x, u, D u, D) M^{k+2} U=P_{1(k+2)}(D) M^{k+2} U+G_{k+2}\left(D M^{k+1} U, D^{k+3} \varphi\right) \text {, }
$$

where $P_{1(k+2)}(D)=P_{1(k+2)}\left(D U, D^{2} \varphi, D\right)$ is first order operator. Consequently, (3.9) may be improved to $M^{k+2} U \in C\left(\mathbf{R} ; H^{s-4}\left(\mathbf{R}^{n}\right)\right) \cap C^{1}\left(\mathbf{R} ; H^{s-5}\left(\mathbf{R}^{n}\right)\right)$. Since 
an equation of the form (3.11) holds for $D^{k+4} \varphi$, with $M D^{3} u$ replaced by $M^{k+2} D^{3} u$

$$
\begin{aligned}
\left(D^{k+4} \varphi\right)_{t}= & F_{1(k+2)}\left(D u\left(t, \varphi, x^{\prime}\right), \tilde{D} \varphi\right) \tilde{D}\left(D^{k+4} \varphi\right) \\
& +F_{2(k+2)}\left(D^{2} u\left(t, \varphi, x^{\prime}\right), D^{2} \varphi\right)\left(D^{k+4} \varphi\right) \\
& +F_{3(k+2)}\left(M^{k+2} D^{3} u\left(t, \varphi, x^{\prime}\right), D^{k+3} \varphi\right),
\end{aligned}
$$

it follows that (3.6) is then improved to $\varphi \in C\left(\mathbf{R} ; H^{s_{0}+k+2}\left(\mathbf{R}^{n-1}\right)\right)$. The induction step is complete, and the regularity of the characteristic hypersurface $\Sigma$ and the conormal regularity of $u$ are established.

\subsection{Conormal regularity with respect to a pair of characteristic hypersurfaces.}

Definition 3.2. Let $\Sigma_{0}$ and $\Sigma_{1}$ be $C^{2}$ characteristic hypersurfaces for $P_{2}$ given in (1.2) intersecting transversally. $w(t, x) \in C\left(\mathbf{R} ; H_{\text {loc }}^{\tau}\left(\mathbf{R}^{n}\right)\right) \cap C^{1}\left(\mathbf{R} ; H_{\text {loc }}^{\tau-1}\left(\mathbf{R}^{n}\right)\right)$ is said to be conormal with respect to $\left\{\Sigma_{0}, \Sigma_{1}\right\}$ if $M_{1} \cdots M_{j} w \in$ $C\left(\mathbf{R} ; H_{\mathrm{loc}}^{\tau}\left(\mathbf{R}^{n}\right)\right) \cap C^{1}\left(\mathbf{R} ; H_{\mathrm{loc}}^{\tau-1}\left(\mathbf{R}^{n}\right)\right)$ for all $C\left(\mathbf{R} ; H_{\mathrm{loc}}^{\tau}\left(\mathbf{R}^{n}\right)\right) \cap C^{1}\left(\mathbf{R} ; H_{\mathrm{loc}}^{\tau-1}\left(\mathbf{R}^{n}\right)\right)$ vector fields $M_{1}, \cdots, M_{j}$ simultaneously tangent to both $\Sigma_{0}$ and $\Sigma_{1}$, written $w \in N^{\tau, \infty}\left(\Sigma_{0}, \Sigma_{1}\right)$. If this property holds for all $j \leq k$, then $u$ is said to be conormal of degree $k$ with respect to $\left\{\Sigma_{0}, \Sigma_{1}\right\}$, written $w \in N^{\tau, k}\left(\Sigma_{0}, \Sigma_{1}\right)$.

Suppose that $\Sigma_{0}$ and $\Sigma_{1}$ are characteristic hypersurfaces for $P_{2}$ given locally by $\left\{x_{1}=\varphi^{0}\left(t, x^{\prime}\right)\right\}$ and $\left\{x_{1}=\varphi^{1}\left(t, x^{\prime}\right)\right\}$ respectively, and suppose that $\Sigma_{0}$ and $\Sigma_{1}$ intersect transversally with $\varphi_{t}^{0}-\varphi_{t}^{1} \neq 0$. Let $\Gamma=\Sigma_{0} \cap \Sigma_{1}$. Suppose that $u \in C\left(\mathbf{R} ; H_{\mathrm{loc}}^{s}\left(\mathbf{R}^{n}\right)\right) \cap C^{1}\left(\mathbf{R} ; H_{\mathrm{loc}}^{s-1}\left(\mathbf{R}^{n}\right)\right), s>\frac{n}{2}+4$. Then $\varphi^{0}$ and $\varphi^{1}$ satisfy (2.22) with coefficients evaluated at $x_{1}=\varphi^{0}\left(t, x^{\prime}\right)$ and $x_{1}=$ $\varphi^{1}\left(t, x^{\prime}\right)$ respectively, and $\varphi^{0}, \varphi^{1} \in C\left(\mathbf{R} ; H_{\mathrm{loc}}^{s-2}\left(\mathbf{R}^{n-1}\right)\right) \cap C^{1}\left(\mathbf{R} ; H_{\mathrm{loc}}^{s-3}\left(\mathbf{R}^{n-1}\right)\right) \subset$ $C^{2}\left(\mathbf{R} \times \mathbf{R}^{n-1}\right)$ by Theorem 2.11 .

Consider a change of variables:

$$
y_{0}=x_{1}-\varphi^{0}\left(t, x^{\prime}\right), y_{1}=x_{1}-\varphi^{1}\left(t, x^{\prime}\right), y_{2}=x_{2}, \cdots, y_{n}=x_{n} .
$$

We know that the Jacobian of (3.12) is $\varphi_{t}^{1}-\varphi_{t}^{0} \neq 0$. Let $\psi\left(t, x^{\prime}\right)=\varphi^{0}\left(t, x^{\prime}\right)-$ $\varphi^{1}\left(t, x^{\prime}\right)$.

Under the change of variables (3.12), $\Sigma_{0}$ and $\Sigma_{1}$ become locally $S_{0}=$ $\left\{y_{0}=0\right\}$ and $S_{1}=\left\{y_{1}=0\right\}$ respectively in the $y$-coordinates. The vector fields tangential to both $S_{0}$ and $S_{1}$ are generated by the vector fields $y_{0} \frac{\partial}{\partial y_{0}}, y_{1} \frac{\partial}{\partial y_{1}}, \frac{\partial}{\partial y_{2}}, \cdots, \frac{\partial}{\partial y_{n}}$. Therefore, by the chain rule, the $C^{2}$ vector fields tangent to both $\Sigma_{0}$ and $\Sigma_{1}$ are spanned by

$$
\begin{aligned}
& M_{0}=-\frac{1}{\psi_{t}}\left(x_{1}-\varphi^{0}\left(t, x^{\prime}\right)\right)\left(\partial_{t}+\varphi_{t}^{1}\left(t, x^{\prime}\right) \partial_{x_{1}}\right), \\
& M_{1}=\frac{1}{\psi_{t}}\left(x_{1}-\varphi^{1}\left(t, x^{\prime}\right)\right)\left(\partial_{t}+\varphi_{t}^{0}\left(t, x^{\prime}\right) \partial_{x_{1}}\right), \\
& M_{j}=\frac{1}{\psi_{t}}\left(-\psi_{x_{j}} \partial_{t}+\left(\varphi_{t}^{0} \varphi_{x_{j}}^{1}-\varphi_{t}^{1} \varphi_{x_{j}}^{0}\right) \partial_{x_{1}}+\psi_{t} \partial_{x_{j}}\right), \quad 2 \leq j \leq n .
\end{aligned}
$$

Let $D_{0}=\frac{1}{\psi_{t}}\left(\partial_{t}+\varphi_{t}^{1} \partial_{x_{1}}\right)$ and $D_{1}=\frac{1}{\psi_{t}}\left(\partial_{t}+\varphi_{t}^{0} \partial_{x_{1}}\right)$. Then

$$
M_{0}=-\left(x_{1}-\varphi^{0}\right) D_{0}, M_{1}=\left(x_{1}-\varphi^{1}\right) D_{1} \quad \text { and } \quad M_{j}=-\varphi_{x_{j}}^{0} D_{0}+\varphi_{x_{j}}^{1} D_{1}+\partial_{x_{j}} \text {. }
$$


Notice that $\partial_{t}=\varphi_{t}^{0} D_{0}-\varphi_{t}^{1} D_{1}, \partial_{x_{1}}=D_{1}-D_{0}$ and $\partial_{x_{j}}=\varphi_{x_{j}}^{0} D_{0}-\varphi_{x_{j}}^{1} D_{1}+M_{j}$. We have the following expressions: For $2 \leq i, j \leq n$,

$$
\begin{aligned}
\partial_{t}^{2}= & \left(\varphi_{t}^{0}\right)^{2} D_{0}^{2}-2 \varphi_{t}^{0} \varphi_{t}^{1} D_{0} D_{1}+\left(\varphi_{t}^{1}\right)^{2} D_{1}^{2}+h_{00}\left(D^{2} \varphi, D\right), \\
\partial_{t} \partial_{x_{1}}= & -\varphi_{t}^{0} D_{0}^{2}+\left(\varphi_{t}^{0}+\varphi_{t}^{1}\right) D_{0} D_{1}-\varphi_{t}^{1} D_{1}^{2}, \\
\partial_{t} \partial_{x_{j}}= & \left(\varphi_{t}^{0} \varphi_{x_{j}}^{0}\right) D_{0}^{2}-\left(\varphi_{t}^{1} \varphi_{x_{j}}^{0}\right) D_{0} D_{1}+\left(\varphi_{t}^{1} \varphi_{x_{j}}^{1}\right) D_{1}^{2} \\
& \quad+\varphi_{t}^{0} D_{0} M_{j}-\varphi_{t}^{1} D_{1} M_{j}+h_{0 j}\left(D^{2} \varphi, D\right), \\
\partial_{x_{1}}^{2}= & D_{0}^{2}-2 D_{0} D_{1}+D_{1}^{2} \\
\partial_{x_{1}} \partial_{x_{j}}= & -\varphi_{x_{j}}^{0} D_{0}^{2}+\left(\varphi_{x_{j}}^{0}+\varphi_{x_{j}}^{1}\right) D_{0} D_{1}-\varphi_{x_{j}}^{1} D_{1}^{2} \\
& \quad+D_{1} M_{j}-D_{0} M_{j}+h_{1 j}\left(D^{2} \varphi, D\right), \\
\partial_{x_{j}}^{2}= & \left(\varphi_{x_{j}}^{0}\right)^{2} D_{0}^{2}-2\left(\varphi_{x_{j}}^{0} \varphi_{x_{j}}^{1}\right) D_{0} D_{1}+\left(\varphi_{x_{j}}^{1}\right)^{2} D_{1}^{2} \\
& \quad+2 \varphi_{x_{j}}^{0} D_{0} M_{j}-2 \varphi_{x_{j}}^{1} D_{1} M_{j}+M_{j}^{2}+h_{j j}\left(D^{2} \varphi, D\right), \\
\partial_{x_{i}} \partial_{x_{j}}= & \left(\varphi_{x_{i}}^{0} \varphi_{x_{j}}^{0}\right) D_{0}^{2}-\left(\varphi_{x_{i}}^{0} \varphi_{x_{j}}^{1}+\varphi_{x_{j}}^{0} \varphi_{x_{i}}^{1}\right) D_{0} D_{1}+\left(\varphi_{x_{i}}^{1} \varphi_{x_{j}}^{1}\right) D_{1}^{2} \\
& \quad+\left(\varphi_{x_{i}}^{0}+\varphi_{x_{j}}^{0}\right) D_{0} M_{j}-\left(\varphi_{x_{i}}^{1}+\varphi_{x_{j}}^{1}\right) D_{1} M_{j}+M_{i} M_{j}+h_{i j}\left(D^{2} \varphi, D\right) .
\end{aligned}
$$

Then

$$
\begin{aligned}
P_{2}(t, x, u, D u, D)= & c_{0} D_{0}^{2}+c_{1} D_{1}^{2}+c_{01} D_{0} D_{1}+\sum_{j=2}^{n} c_{0 j} D_{0} M_{j} \\
& +\sum_{j=2}^{n} c_{1 j} D_{1} M_{j}+\sum_{i, j=2}^{n} c_{i j} M_{i} M_{j}+h(D),
\end{aligned}
$$

where

$$
c_{k}=\left(\varphi_{t}^{k}\right)^{2}-a_{11}+2\left(a_{01}-\sum_{j=2}^{n} a_{0 j}\right) \varphi_{t}^{k}+2 \sum_{j=2}^{n} a_{1 j} \varphi_{x_{j}}^{k}-\sum_{i, j=2}^{n} a_{i j} \varphi_{x_{i}}^{k} \varphi_{x_{j}}^{k}
$$

for $k=0,1, c_{i j}=c_{i j}\left(D^{2} u, D \varphi\right)$ and $h(D)=h\left(D^{2} u, D^{2} \varphi, D\right)$ is a first order operator. Let, for $k=0,1$,

$$
F_{k}\left(t, x_{1}, x^{\prime}\right)=\left(\varphi_{t}^{k}\right)^{2}-a_{11}+2\left(a_{01}-\sum_{j=2}^{n} a_{0 j}\right) \varphi_{t}^{k}+2 \sum_{j=2}^{n} a_{1 j} \varphi_{x_{j}}^{k}-\sum_{i, j=2}^{n} a_{i j} \varphi_{x_{i}}^{k} \varphi_{x_{j}}^{k} .
$$

Since $\Sigma_{0}$ and $\Sigma_{1}$ are characteristic hypersurfaces for $P_{2}, F_{k}\left(t, \varphi^{k}\left(t, x^{\prime}\right), x^{\prime}\right) \equiv$ 0 . Therefore, as we see in the proof of Theorem 3.2,

$$
F_{k}\left(t, x_{1}, x^{\prime}\right)=\left(x_{1}-\varphi^{k}\right) \tilde{F}_{k}\left(t, x_{1}, x^{\prime}\right),
$$

where $\tilde{F}_{k}$ is an integral of a smooth function of $t, x^{\prime}, u, D u, D^{2} u, \varphi$ and $\tilde{D} \varphi$. Therefore, $c_{0}=c_{0}\left(D^{2} u, D \varphi\right)\left(x_{1}-\varphi^{0}\right)$ and $c_{1}=c_{1}\left(D^{2} u, D \varphi\right)\left(x_{1}-\varphi^{1}\right)$. By the assumption of strictly hyperbolicity, $c_{01} \neq 0$, so after division we assume 
that $c_{01} \equiv 1$. Therefore,

$$
\begin{aligned}
P_{2}(t, x, u, D u, D)= & D_{0} D_{1}+r_{00} D_{0} M_{0}+r_{11} D_{1} M_{1}+\sum_{j=2}^{n} r_{0 j} D_{0} M_{j} \\
& +\sum_{j=2}^{n} r_{1 j} D_{1} M_{j}+\sum_{i, j=2}^{n} r_{i j} M_{i} M_{j}+b(D),
\end{aligned}
$$

where $r_{i j}=r_{i j}\left(D^{2} u, D \varphi\right)$ and $b(D)=b\left(D^{2} u, D^{2} \varphi, D\right)$ is a first order operator.

Before we consider commutators $\left[P_{2}, M_{j}\right]$, for $j=0,1, \cdots, n$, we will first observe several basic commutators. Let $\tilde{D}_{0}=\partial_{t}+\varphi_{t}^{1} \partial_{x_{1}}$ and $\tilde{D}_{1}=\partial_{t}+$ $\varphi_{t}^{0} \partial_{x_{1}}$. Since we have $\left[\tilde{D}_{0}, \tilde{D}_{1}\right]=\psi_{t t} \partial_{x_{1}}$ and $\left(\tilde{D}_{0}\left(1 / \psi_{t}\right)\right) \tilde{D}_{1}-\left(\tilde{D}_{1}\left(1 / \psi_{t}\right)\right) \tilde{D}_{0}=$ $-\left(\psi_{t t} /\left(\psi_{t}\right)^{2}\right) \partial_{x_{1}},\left[D_{0}, D_{1}\right]=0$. Therefore, we have the following lemma.

Lemma 3.3. Let $D_{0}$ and $D_{1}$ be first order operators as above. Then $D_{0}\left(x_{1}-\varphi^{0}\right)=-1, D_{0}\left(x_{1}-\varphi^{1}\right)=0, D_{1}\left(x_{1}-\varphi^{0}\right)=0$, and $D_{1}\left(x_{1}-\varphi^{1}\right)=1$.

By using Lemma 3.3, we can compute commutators $\left[D_{j}, M_{j}\right]$ for $i=0,1$ and $j=0,1, \cdots, n$.

Lemma 3.4. Let $D_{0}, D_{1}$ and $M_{j}$ be as above. Then we have

$$
\left[D_{0}, M_{0}\right]=D_{0},\left[D_{0}, M_{1}\right]=\left[D_{1}, M_{0}\right]=0,\left[D_{1}, M_{1}\right]=D_{1}
$$

and

$$
\left[D_{0}, M_{j}\right]=\left[D_{1}, M_{j}\right]=0 \text { for } j=2, \cdots, n \text {. }
$$

Proof. We consider only $\left[D_{0}, M_{j}\right]$ and $\left[D_{1}, M_{j}\right]$. We note that $\left[D_{0}, \partial_{x_{j}}\right]=$ $\left[D_{1}, \partial_{x_{j}}\right]=\left(1 / \psi_{t}\right)\left(\varphi_{t x_{j}}^{0} D_{0}-\varphi_{t x_{j}}^{1} D_{1}\right), D_{0}\left(\varphi_{x_{j}}^{0}\right)=D_{1}\left(\varphi_{x_{j}}^{0}\right)=\left(1 / \psi_{t}\right) \varphi_{t x_{j}}^{0}$ and $D_{0}\left(\varphi_{x_{j}}^{1}\right)=D_{1}\left(\varphi_{x_{j}}^{1}\right)=\left(1 / \psi_{t}\right) \varphi_{t x_{j}}^{1}$. Therefore, for $i=0,1$ and $j=2, \cdots, n$,

$$
\left[D_{i}, M_{j}\right]=\left[D_{i},-\varphi_{x_{j}}^{0} D_{0}+\varphi_{x_{j}}^{1} D_{1}+\partial_{x_{j}}\right]=0 .
$$

This completes the proof of lemma.

For the commutators $\left[M_{i}, M_{j}\right]$, for $i, j=0,1, \cdots, n$, we have the following lemma.

Lemma 3.5. Let $M_{0}, M_{1}, \cdots, M_{n}$ be as above. Then, for $i, j=2, \cdots, n$ with $i \neq j$, we have

$$
\left[M_{0}, M_{1}\right]=\left[M_{0}, M_{j}\right]=\left[M_{1}, M_{j}\right]=\left[M_{i}, M_{j}\right]=0 .
$$

Proof. We can easily see, from Lemma 3.3, that $\left[M_{0}, M_{1}\right]=0$. Notice that

$$
-\left(M_{0}\left(\varphi_{x_{j}}^{0}\right)\right) D_{0}+\left(M_{0}\left(\varphi_{x_{j}}^{1}\right)\right) D_{1}-\left(x_{1}-\varphi^{0}\right)\left[D_{0}, \partial_{x_{j}}\right]=0
$$

and

$$
-\varphi_{x_{j}}^{0}\left[M_{0}, D_{0}\right]+\left(\partial_{x_{j}}\left(x_{1}-\varphi^{0}\right)\right) D_{0}=0
$$

Similarly, we have

$$
-\left(M_{1}\left(\varphi_{x_{j}}^{0}\right)\right) D_{0}+\left(M_{1}\left(\varphi_{x_{j}}^{1}\right)\right) D_{1}+\left(x_{1}-\varphi^{1}\right)\left[D_{0}, \partial_{x_{j}}\right]=0
$$


and

$$
\varphi_{x_{j}}^{1}\left[M_{1}, D_{1}\right]-\left(\partial_{x_{j}}\left(x_{1}-\varphi^{1}\right)\right) D_{1}=0 .
$$

Therefore, $\left[M_{0}, M_{j}\right]=\left[M_{1}, M_{j}\right]=0$ for $j=2, \cdots, n$. Since

$$
\begin{aligned}
{\left[M_{i}, M_{j}\right]=} & {\left[-\varphi_{x_{i}}^{0} D_{0}+\varphi_{x_{i}}^{1} D_{1}+\partial_{x_{i}}, M_{j}\right] } \\
= & -\varphi_{x_{i}}^{0}\left[D_{0}, M_{j}\right]+\left(M_{j}\left(\varphi_{x_{i}}^{0}\right)\right) D_{0}+\varphi_{x_{i}}^{1}\left[D_{1}, M_{j}\right] \\
& -\left(M_{j}\left(\varphi_{x_{i}}^{1}\right)\right) D_{1}+\left[\partial_{x_{i}}, M_{j}\right],
\end{aligned}
$$

by Lemma 3.4, it is enough to show that the coefficient of $D_{0}$ and $D_{1}$ are 0 . We note that $D_{0}\left(\varphi_{x_{i}}^{0}\right)=D_{1}\left(\varphi_{x_{i}}^{0}\right)=\left(1 / \psi_{t}\right) \varphi_{t x_{i}}^{0}$ and $D_{0}\left(\varphi_{x_{i}}^{1}\right)=D_{1}\left(\varphi_{x_{i}}^{1}\right)=$ $\left(1 / \psi_{t}\right) \varphi_{t x_{i}}^{1}$. From the note above, the coefficient of $D_{0}$, which comes from the terms $\left(M_{j}\left(\varphi_{x_{i}}^{0}\right)\right) D_{0}$ and $\left[\partial_{x_{i}}, M_{j}\right]$, is 0 . Similarly, the coefficients of $D_{1}$ becomes 0 . Therefore, $\left[M_{i}, M_{j}\right]=0$.

Lemma 3.6. Let $D_{0}, D_{1}$ and $M_{j}$ be as above. Then we have

$$
\begin{aligned}
& {\left[D_{0} D_{1}, M_{0}\right]=D_{0} D_{1},\left[D_{0} D_{1}, M_{0}\right]=D_{0} D_{1},} \\
& {\left[D_{0} D_{1}, M_{k}\right]=0 \text { for } k=2, \cdots, n,} \\
& {\left[D_{0} M_{j}, M_{0}\right]=D_{0} M_{j},\left[D_{0} M_{j}, M_{k}\right]=0 \text { for } k \neq 0,} \\
& {\left[D_{1} M_{j}, M_{1}\right]=D_{1} M_{j},\left[D_{1} M_{j}, M_{k}\right]=0 \text { for } k \neq 1}
\end{aligned}
$$

and

$$
\left[M_{i} M_{j}, M_{k}\right]=0 \quad \text { for } i, j, k=0,1, \cdots, n .
$$

Proof. The proof immediately follows from Lemma 3.4, Lemma 3.5 and the fact that $[A B, C]=A[B, C]+[A, C] B$.

From Lemma 3.6, we have the following lemma.

Lemma 3.7. Let $P_{2}(t, x, u, D u, D)$ be a strictly hyperbolic quasilinear operator given in (1.2). Let $\Sigma_{0}$ and $\Sigma_{1}$ be characteristic hypersurfaces for $P_{2}$ intersecting transversally. Suppose that $\Sigma_{0}$ and $\Sigma_{1}$ are given locally by $\left\{x_{1}=\varphi^{0}\left(t, x^{\prime}\right)\right\}$ and $\left\{x_{1}=\varphi^{1}\left(t, x^{\prime}\right)\right\}$ respectively, with $\varphi^{0}, \varphi^{1} \in C^{2}\left(\mathbf{R} \times \mathbf{R}^{n-1}\right)$ and $\psi_{t} \neq 0$. Let $u \in C\left(\mathbf{R} ; H_{\text {loc }}^{s}\left(\mathbf{R}^{n}\right)\right) \cap C^{1}\left(\mathbf{R} ; H_{\text {loc }}^{s-1}\left(\mathbf{R}^{n}\right)\right), s>\frac{n}{2}+4$. Then the $C^{2}$ vector fields tangent to both $\Sigma_{0}$ and $\Sigma_{1}$ are spanned by $M_{0}, M_{1}, \cdots, M_{n}$, and

$$
\left[P_{2}, M_{j}\right]=P_{2}+\sum_{i=0}^{n} r_{i j}(D) M_{i}+h_{j}(D), \quad \text { for } j=0,1 \text {, }
$$

and

$$
\left[P_{2}, M_{j}\right]=\sum_{i=0}^{n} r_{i j}(D) M_{i}+h_{j}(D), \quad \text { for } j=2, \cdots, n \text {, }
$$

where $r_{i j}(D)=r_{i j}\left(D^{3} u, D^{2} \varphi^{0}, D^{2} \varphi^{1}, D\right), h_{j}(D)=h_{j}\left(D^{3} u, D^{3} \varphi^{0}, D^{3} \varphi^{1}, D\right)$ are first order operators.

Proof. The proof follows from Lemma 3.16 and the fact that $[a A, B]=a[A, B]$ $-(B a) A$.

Now, we can state and prove the conormal regularity of a solution of equation (1.1) with respect to a pair of characteristic hypersurfaces intersecting transversally. 
Theorem 3.8. Let $P_{2}$ be as above and let $\Sigma_{0}$ and $\Sigma_{1}$ be characteristic hypersurfaces for $P_{2}$ intersecting transversally in $\{t \geq 0\}$. Suppose that $u \in$ $C\left(\mathbf{R} ; H_{\mathrm{loc}}^{s}\left(\mathbf{R}^{n}\right)\right) \cap C^{1}\left(\mathbf{R} ; H_{\mathrm{loc}}^{s-1}\left(\mathbf{R}^{n}\right)\right), s>\frac{n}{2}+4$, and $u$ satisfies the equation

$$
P_{2}(t, x, u, D u, D) u=f(t, x, u, D u),
$$

where $f$ is a smooth function of its arguments. If $\Sigma_{0}$ and $\Sigma_{1}$ are smooth in $\{t<0\}$, and $u \in N^{s, \infty}\left(\Sigma_{0}, \Sigma_{1}\right)$ in $\{t<0\}$, then we have:

(1) $\Gamma$ is smooth,

(2) $\Sigma_{0}$ and $\Sigma_{1}$ are smooth outside $\Gamma$,

(3) $u$ is smooth outside $\Sigma_{0} \cup \Sigma_{1}$,

(4) locally, $u \in N^{s, \infty}\left(\Sigma_{0}, \Sigma_{1}\right)$.

Proof. By finite propagation speed and an analysis local in time, it may be assumed that $u$ has compact support in $x$ for each $t$, and that $\Sigma_{0}$ and $\Sigma_{1}$ are given by $\left\{x_{1}=\varphi^{0}\left(t, x^{\prime}\right)\right\}$ and $\left\{x_{1}=\varphi^{1}\left(t, x^{\prime}\right)\right\}$ respectively. Assume that $\psi_{t} \neq 0$. Then $\varphi^{0}$ and $\varphi^{1}$ satisfy (2.22). From now on, as a matter of notational convenience, we use $\varphi$ for both $\varphi^{0}$ and $\varphi^{1}$. As we see in the proof of Theorem 3.2, $\varphi \in C^{2}\left(\mathbf{R} \times \mathbf{R}^{n-1}\right)$ and for $s_{0}=s-2$,

$$
\varphi\left(t, x^{\prime}\right) \in C\left(\mathbf{R} ; H^{s_{0}}\left(\mathbf{R}^{n-1}\right)\right)
$$

since $s>\frac{n}{2}+4$.

Let $U$ stand for the vector of all derivatives of $u$ up to fourth order. From (3.16) and the assumption on $u, U \in C\left(\mathbf{R} ; H^{s-4}\left(\mathbf{R}^{n}\right)\right) \cap C^{1}\left(\mathbf{R} ; H^{s-5}\left(\mathbf{R}^{n}\right)\right)$ and

$$
P_{2}(t, x, u, D u, D) U=g(D U),
$$

where the right-hand side is meaningful as we noted in the proof of Theorem 3.2. Let $M$ stand for the vector operator $\left(1, M_{0}, M_{1}, \cdots, M_{n}\right)$. We note that $\left[M_{i}, D\right]=h_{i}\left(D^{2} \varphi, D\right)$. From (3.18) and Lemma 3.7,

$$
P_{2}(t, x, u, D u, D) M U=\left[P_{2}, M\right] U+M(g(D U))
$$

$$
\begin{aligned}
& =P_{2} U+c(D) M U+d(D) U+(M D U) g^{\prime}(D U) \\
& =P_{1}(D) M U+G(D U),
\end{aligned}
$$

where $G(D U)=G\left(D U, D^{3} \varphi\right)$ and $c(D)=c\left(D^{3} u, D^{2} \varphi, D\right), d(D)=$ $d\left(D^{3} u, D^{3} \varphi, D\right)$ and $P_{1}(D)=P_{1}\left(D U, D^{2} \varphi, D\right)$ are first order operators.

The coefficients of $P_{2}$ are in $C\left(\mathbf{R} ; H^{s-1}\left(\mathbf{R}^{n}\right)\right) \cap C^{1}\left(\mathbf{R} ; H^{s-2}\left(\mathbf{R}^{n}\right)\right)$, while by (3.17) and (2.22), $G(D U)$ and the coefficients of $P_{1}\left(D U, D^{2} \varphi, D\right)$ are in the space $C\left(\mathbf{R} ; H^{s-5}\left(\mathbf{R}^{n}\right)\right)$. It then follows from (3.19) and Corollary 2.6 that

$$
M U \in C\left(\mathbf{R} ; H^{s-4}\left(\mathbf{R}^{n}\right)\right) \cap C^{1}\left(\mathbf{R} ; H^{s-5}\left(\mathbf{R}^{n}\right)\right) .
$$

In order to use the improved regularity of $U$, it is convenient to differentiate (2.22) two times. It follows that there are smooth functions $f_{j}$ such that

$$
\begin{aligned}
\left(D^{2} \varphi\right)_{t}= & f_{1}\left(D u\left(t, \varphi, x^{\prime}\right), \tilde{D} \varphi\right) \tilde{D}\left(D^{2} \varphi\right)+f_{2}\left(D^{2} u\left(t, \varphi, x^{\prime}\right), D^{2} \varphi\right) \\
& +f_{3}\left(D^{3} u\left(t, \varphi, x^{\prime}\right), D \varphi\right),
\end{aligned}
$$

where $\tilde{D}$ is the vector operator $\left(\partial_{x_{2}}, \cdots, \partial_{x_{n}}\right)$.

Let $\phi_{j}=\varphi_{t}^{0} \varphi_{x_{j}}^{1}-\varphi_{t}^{1} \varphi_{x_{j}}^{0}, \mathscr{L}^{0}=L_{1}^{0}, L_{j}^{0}$ and $\mathscr{L}^{1}=L_{1}^{1}, L_{j}^{1}$, where $L_{1}^{0}=$ $\left(\psi / \psi_{t}\right)\left(\partial_{t}+\varphi_{t}^{0} \partial_{x_{1}}\right), L_{1}^{1}=\left(\psi / \psi_{t}\right)\left(\partial_{t}+\varphi_{t}^{1} \partial_{x_{1}}\right)$ and

$$
L_{j}^{0}=L_{j}^{1}=\left(1 / \psi_{t}\right)\left(-\psi_{x_{j}} \partial_{t}+\phi_{j} \partial_{x_{1}}+\psi_{t} \partial_{x_{j}}\right) .
$$


We note that $[L, D]=k\left(D^{2} \psi, \partial_{t}, \partial_{x_{j}}\right)+k\left(D^{2} \psi, D^{2} \varphi, \partial_{x_{1}}\right)$ for $L \in \mathscr{L}^{0} \cup \mathscr{L}^{1}$. Since $\partial_{t}=\left(\psi_{t} / \psi\right) \tilde{L}_{1}$ and $\partial_{x_{j}}=\left(\psi_{x_{j}} / \psi\right) \tilde{L}_{1}-\tilde{L}_{j}$ away from $\psi=0$, where $\tilde{L}_{1}=\left(\psi / \psi_{t}\right) \partial_{t}$ and $\tilde{L}_{j}=\left(1 / \psi_{t}\right)\left(-\psi_{x_{j}} \partial_{t}+\psi_{t} \partial_{x_{j}}\right), D\left(D^{2} \varphi\right)=l(D \psi)\left(\tilde{L} D^{2} \varphi\right)$ away from $\{\psi=0\}$.

Since $(L v)\left(t, \varphi^{i}, x^{\prime}\right)=L\left(v\left(t, \varphi^{i}, x^{\prime}\right)\right)$ for $L \in \mathscr{L}^{i}, i=0,1$, it follows from applying $L \in \mathscr{L}^{i}$ to (3.21) with $\varphi^{i}$ in place of $\varphi$ respectively, that there are smooth functions $F_{j}$ away from $\psi=0$ such that

$$
\begin{aligned}
\left(\tilde{L} D^{2} \varphi^{i}\right)_{t}= & F_{1}\left(D u\left(t, \varphi^{i}, x^{\prime}\right), \tilde{D} \varphi^{i}\right) \tilde{D}\left(\tilde{L} D^{2} \varphi^{i}\right) \\
& +F_{2}\left(D^{2} u\left(t, \varphi^{i}, x^{\prime}\right), D^{2} \varphi^{i}, D^{2} \psi\right)\left(\tilde{L} D^{2} \varphi^{i}\right) \\
& +F_{3}\left(L D^{3} u\left(t, \varphi^{i}, x^{\prime}\right), D^{2} \varphi^{i}, D \psi\right) .
\end{aligned}
$$

Since $D M D^{3} u=M D^{4} u+[D, M] D^{3} u \in C\left(\mathbf{R} ; H^{s-4}\left(\mathbf{R}^{n}\right)\right)$, we have $M D^{3} u \in$ $C\left(\mathbf{R} ; H^{s-3}\left(\mathbf{R}^{n}\right)\right)$. By Corollary $2.12, L D^{3} u\left(t, \varphi^{i}, x^{\prime}\right) \in C\left(\mathbf{R} ; H^{s-4}\left(\mathbf{R}^{n-1}\right)\right)$. Therefore, Corollary 2.12 implies that $\tilde{L} D^{2} \varphi \in C\left(\mathbf{R} ; H^{s_{0}-2}\left(\mathbf{R}^{n-1}\right)\right)$.

The functions $\varphi^{i}$ (and hence $\psi$ ) are not necessarily smooth across $\left\{\varphi^{0}=\right.$ $\left.\varphi^{1}\right\}$ (that is, $\{\psi=0\}$ ), as is known from the example of Messer [9]. On the other hand, $\Gamma$ itself (defined by $\{\psi=0\} \cap\left\{x_{1}=\varphi^{0}\right\}$ ) will be seen to be smooth. In order to avoid the functions $\varphi^{i}$ on the set $\{\psi=0\} \cap\left\{x_{1}=\varphi^{0}\right\}$, we use finite propagation speed and analyze regularity separately in the past and in the future.

Since $\psi_{t} \neq 0$, by the implicit function theorem, there is a function $t=\tau\left(x^{\prime}\right)$ such that $\psi\left(\tau\left(x^{\prime}\right), x^{\prime}\right) \equiv 0$. Therefore, $\Gamma$ can be expressed by $\left\{\left(t, x_{1}, x^{\prime}\right)\right.$ : $\left.\psi\left(\tau\left(x^{\prime}\right), x^{\prime}\right)=0\right\} \cap\left\{\left(t, x_{1}, x^{\prime}\right): x_{1}=\varphi^{0}\left(\tau\left(x^{\prime}\right), x^{\prime}\right)\right\}$. For sufficiently small $\epsilon>0$, let

$$
\begin{aligned}
& R_{1}=\left\{\left(t, x_{1}, x^{\prime}\right): t+\epsilon\left|x_{1}-\varphi^{0}\left(\tau\left(x^{\prime}\right), x^{\prime}\right)\right|^{2}<\tau\left(x^{\prime}\right)\right\}, \\
& R_{2}=\left\{\left(t, x_{1}, x^{\prime}\right): t+\epsilon\left|x_{1}-\varphi^{0}\left(\tau\left(x^{\prime}\right), x^{\prime}\right)\right|^{2}>\tau\left(x^{\prime}\right)\right\}, \\
& R_{3}=\mathbf{R}^{n+1} \backslash\left(R_{1} \cup R_{2}\right) .
\end{aligned}
$$

On $R_{3}, u$ is smooth away from $\Gamma$ by finite propagation speed. Therefore, we will consider the lower region $R_{1}$ and upper region $R_{2}$. Let

$$
M_{j}^{R_{i}}= \begin{cases}M_{j} & \text { on } R_{i} \text { for } \mathrm{i}=1,2 \\ 0 & \text { otherwise }\end{cases}
$$

Then we have Lemma 3.7, (3.19), (3.20) and (3.22) for $M_{j}^{R_{i}}$. Applying $M^{R_{i}}$ to equation (3.19), we obtain the equation

$$
P_{2}\left(M^{R_{i}}\right)^{2} U=P_{12}(D)\left(M^{R_{i}}\right)^{2} U+G_{2}\left(D M^{R_{i}} U, M^{R_{i}} D^{2} \varphi\right) .
$$

From (3.22), $M^{R_{i}} D^{2} \varphi \in C\left(\mathbf{R} ; H^{s_{0}-2}\left(\mathbf{R}^{n-1}\right)\right)$ and then, by Corollary 2.12,

$$
\left(M^{R_{i}}\right)^{2} U \in C\left(\mathbf{R} ; H^{s-4}\left(\mathbf{R}^{n}\right)\right) \cap C^{1}\left(\mathbf{R} ; H^{s-5}\left(\mathbf{R}^{n}\right)\right) .
$$

Applying $L \in \mathscr{L}^{i}$ to (3.22), we have the equation

$$
\begin{aligned}
\left(\tilde{L}^{2} D^{2} \varphi^{i}\right)_{t}= & F_{12}\left(D u\left(t, \varphi^{i}, x^{\prime}\right), \tilde{D} \varphi^{i}\right) \tilde{D}\left(\tilde{L}^{2} D^{2} \varphi^{i}\right) \\
& +F_{22}\left(L D^{2} u\left(t, \varphi^{i}, x^{\prime}\right), \tilde{L} D^{2} \varphi^{i}, D^{2} \psi\right)\left(\tilde{L}^{2} D^{2} \varphi^{i}\right) \\
& +F_{32}\left(L^{2} D^{3} u\left(t, \varphi^{i}, x^{\prime}\right), \tilde{L} D^{2} \varphi^{i}, \tilde{L} D^{2} \psi\right)
\end{aligned}
$$


A similar argument as above implies that $\tilde{L}^{2} D^{2} \varphi \in C\left(\mathbf{R} ; H^{s_{0}-2}\left(\mathbf{R}^{n-1}\right)\right)$.

By differentiating $\psi\left(\tau\left(x^{\prime}\right), x^{\prime}\right) \equiv 0$ with respect to $x_{j}$, we have

$$
\psi_{t}\left(\tau\left(x^{\prime}\right), x^{\prime}\right) \frac{\partial \tau}{\partial x_{j}}+\psi_{x_{j}}\left(\tau\left(x^{\prime}\right), x^{\prime}\right)=0 \quad \text { or } \quad \frac{\partial \tau}{\partial x_{j}}=-\frac{\psi_{x_{j}}\left(\tau\left(x^{\prime}\right), x^{\prime}\right)}{\psi_{t}\left(\tau\left(x^{\prime}\right), x^{\prime}\right)}
$$

Therefore, the regularity of $\Gamma$ depends only on the regularity of $\varphi$ since $\tau\left(x^{\prime}\right) \epsilon$ $C^{2}\left(\mathbf{R}^{n-1}\right)$ and $\psi=\varphi^{0}-\varphi^{1}$. More precisely, we have the following:

On $\Gamma$, the vector fields $M_{2}, \cdots, M_{n}$ are equivalent to the vector fields $\partial_{x_{2}}, \cdots, \partial_{x_{n}}$ and, from (3.25),

$$
\begin{aligned}
\partial_{x_{j}}\left(\varphi\left(\tau\left(x^{\prime}\right), x^{\prime}\right)\right) & =\frac{\partial \varphi}{\partial t}\left(\tau\left(x^{\prime}\right), x^{\prime}\right) \frac{\partial \tau}{\partial x_{j}}+\frac{\partial \varphi}{\partial x_{j}}\left(\tau\left(x^{\prime}\right), x^{\prime}\right) \\
& =-\frac{\psi_{x_{j}}}{\psi_{t}}\left(\tau\left(x^{\prime}\right), x^{\prime}\right) \partial_{t} \varphi\left(\tau\left(x^{\prime}\right), x^{\prime}\right)+\partial_{x_{j}} \varphi\left(\tau\left(x^{\prime}\right), x^{\prime}\right) \\
& =\left(M_{j} \varphi\right)\left(\tau\left(x^{\prime}\right), x^{\prime}\right) .
\end{aligned}
$$

Therefore, the improved regularity of $\varphi$ with respect to $M_{2}, \cdots, M_{n}$ implies the improved regularity of $\varphi\left(\tau\left(x^{\prime}\right), x^{\prime}\right)$ with respect to $\partial_{2}, \cdots, \partial_{n}$ and of $\tau\left(x^{\prime}\right)$ with respect to $\partial_{2}, \cdots, \partial_{n}$ since we can write $(3.25)$ as

$$
\frac{\partial \tau}{\partial x_{j}}=-\frac{\psi_{x_{j}}\left(\tau\left(x^{\prime}\right), x^{\prime}\right)}{\psi_{t}\left(\tau\left(x^{\prime}\right), x^{\prime}\right)}=F\left(\varphi_{x_{j}}\left(\tau\left(x^{\prime}\right), x^{\prime}\right), \varphi_{t}\left(\tau\left(x^{\prime}\right), x^{\prime}\right)\right),
$$

where $F$ is a smooth function of its arguments. Moreover, from (3.26),

$$
\begin{aligned}
\frac{\partial^{2} \tau}{\partial_{x_{i}} \partial_{x_{j}}} & =\frac{1}{\psi_{t}^{2}}\left[\left(-\psi_{t x_{j}} \frac{\partial \tau}{\partial x_{i}}-\psi_{x_{i} x_{j}}\right) \psi_{t}+\psi_{x_{j}}\left(\psi_{t t} \frac{\partial \tau}{\partial x_{i}}+\psi_{t x_{i}}\right)\right] \\
& =\frac{1}{\psi_{t}^{3}}\left(\psi_{t x_{j}} \psi_{x_{j}} \psi_{t}-\psi_{x_{i} x_{j}} \psi_{t} \psi_{t}-\psi_{x_{j}} \psi_{t t} \psi_{x_{i}}+\psi_{t x_{i}} \psi_{t} \psi_{x_{j}}\right),
\end{aligned}
$$

evaluated at $\left(\tau\left(x^{\prime}\right), x^{\prime}\right)$, and

$$
\begin{aligned}
\partial_{x_{i}} & \left(\partial_{x_{j}}\left(\varphi\left(\tau\left(x^{\prime}\right), x^{\prime}\right)\right)\right) \\
& =\left(\varphi_{t t} \frac{\partial \tau}{\partial x_{i}}+\varphi_{t x_{i}}\right) \frac{\partial \tau}{\partial x_{j}}+\varphi_{t} \frac{\partial^{2} \tau}{\partial x_{i} \partial x_{j}}+\varphi_{t x_{j}} \frac{\partial \tau}{\partial x_{i}}+\varphi_{x_{i} x_{j}} \\
& =\left(M_{i} M_{j} \varphi\right)\left(\tau\left(x^{\prime}\right), x^{\prime}\right) .
\end{aligned}
$$

Suppose inductively that (3.17) is improved to $\tilde{L}^{k} D^{2} \varphi^{i} \in C\left(\mathbf{R} ; H^{s_{0}-2}\left(\mathbf{R}^{n-1}\right)\right)$ and (3.23) is improved to $\left(M^{R_{i}}\right)^{k+1} U \in C\left(\mathbf{R} ; H^{s-4}\left(\mathbf{R}^{n}\right)\right) \cap C^{1}\left(\mathbf{R} ; H^{s-5}\left(\mathbf{R}^{n}\right)\right)$. From the analogue of (3.24) for $\tilde{L}^{k+1} D^{2} \varphi^{i}$ and Corollary 2.12, it follows that, on each $R_{i}$,

$$
\varphi \in C\left(\mathbf{R} ; H^{s_{0}+k+1}\left(\mathbf{R}^{n-1}\right)\right) \cap \cdots \cap C^{k+1}\left(\mathbf{R} ; H^{s_{0}}\left(\mathbf{R}^{n-1}\right)\right),
$$

since $\partial_{t}=\left(\psi_{t} / \psi\right) \tilde{L}_{1}$ and $\partial_{x_{j}}=\left(\psi_{x_{j}} / \psi\right) \tilde{L}_{1}-\tilde{L}_{j}$. Then, an equation similar to (3.19) holds for $\left(M^{R_{i}}\right)^{k+2} U$ with coefficients depending smoothly on $D\left(M^{R_{i}}\right)^{k+1} U$ and $\left(M^{R_{i}}\right)^{k+1} D^{2} \varphi$, that is,

$$
\begin{aligned}
& P_{2}(t, x, u, D u, D)\left(M^{R_{i}}\right)^{k+2} U \\
& \quad=P_{1(k+2)}(D)\left(M^{R_{i}}\right)^{k+2} U+G_{k+2}\left(D\left(M^{R_{i}}\right)^{k+1} U,\left(M^{R_{i}}\right)^{k+1} D^{2} \varphi\right),
\end{aligned}
$$


where $P_{1(k+2)}(D)=P_{1(k+2)}\left(D U, D^{2} \varphi, D\right)$ is a first order operator. Consequently, (3.20) may be improved to $\left(M^{R_{i}}\right)^{k+2} U \in C\left(\mathbf{R} ; H^{s-4}\left(\mathbf{R}^{n}\right)\right) \cap$ $C^{1}\left(\mathbf{R} ; H^{s-5}\left(\mathbf{R}^{n}\right)\right)$. Since an equation of the form (3.24) holds for $\tilde{L}^{k+2} D^{2} \varphi^{i}$, that is,

$$
\begin{aligned}
\left(\tilde{L}^{k+2} D^{2} \varphi^{i}\right)_{t}= & F_{1(k+2)}\left(D u\left(t, \varphi^{i}, x^{\prime}\right), \tilde{D} \varphi\right) \tilde{D}\left(\tilde{L}^{k+2} D^{2} \varphi^{i}\right) \\
& +F_{2(k+2)}\left(L^{k+1} D u\left(t, \varphi^{i}, x^{\prime}\right), \tilde{L} D \varphi^{i}, D^{2} \psi\right)\left(\tilde{L}^{k+2} D^{2} \varphi^{i}\right) \\
& +F_{3(k+2)}\left(L^{k+2} D^{3} u\left(t, \varphi^{i}, x^{\prime}\right), \tilde{L}^{k+1} D^{2} \varphi^{i}, \tilde{L}^{k+1} D^{2} \psi\right),
\end{aligned}
$$

it follows that (3.17) is then improved to $\varphi \in C\left(\mathbf{R} ; H^{s_{0}+k+2}\left(\mathbf{R}^{n-1}\right)\right)$ on each $R_{i}$. At the same time, the regularity of $\Gamma$ is improved along with the regularity of $\varphi$ by (3.27). The induction step is complete. Therefore, the regularity of $\Gamma$ and the characteristic hypersurfaces $\Sigma_{0}, \Sigma_{1}$ away from $\Gamma$, and the conormal regularity of $u$ are established.

Lemma 3.9. Let $P_{2}$ be as above and $v(t, x)$ satisfy the equation $P_{2} v(t, x)=$ $g(D v)$, where $g$ is a smooth function of its arguments. Let, for all $k_{1}, \cdots, k_{n}$ and for $s>\frac{n}{2}+4$,

$$
\begin{aligned}
& \left(x_{1} \partial_{x_{1}}\right)^{k_{1}}\left(\partial_{x_{2}}\right)^{k_{2}} \cdots\left(\partial_{x_{n}}\right)^{k_{n}} v(0, x) \in H^{s-4}\left(\mathbf{R}^{n}\right), \\
& \left(x_{1} \partial_{x_{1}}\right)^{k_{1}}\left(\partial_{x_{2}}\right)^{k_{2}} \cdots\left(\partial_{x_{n}}\right)^{k_{n}} v_{t}(0, x) \in H^{s-5}\left(\mathbf{R}^{n}\right) .
\end{aligned}
$$

Then, for any $k>0, x_{1}^{k} \partial_{t}^{k} v(0, x), x_{1}^{k} \partial_{x_{1}}^{k} v(0, x), \sum_{l_{1}+l_{2}=k} x_{1}^{k} \partial_{x_{t}}^{l_{1}} \partial_{x_{j}^{2}}^{l_{2}} v(0, x)$ and $\sum_{l_{1}+l_{2}=k} x_{1}^{k} \partial_{x_{1}^{1}}^{l_{1}} \partial_{x_{j}}^{l_{2}} v(0, x)$, where $l_{1}>0$ and $j>0$, are in the space $H^{s-4}\left(\mathbf{R}^{n}\right)$.

Proof. We prove this by induction on $k$. For $k=1, x_{1} \partial_{t} v(0, x) \in H^{s-4}\left(\mathbf{R}^{n}\right)$ since $\partial_{x_{1}}\left(x_{1} \partial_{t}\right) v(0, x)=\partial_{t} v(0, x)+\left(x_{1} \partial_{x_{1}}\right) v_{t}(0, x)$ and $\partial_{x_{j}}\left(x_{1} \partial_{t}\right) v(0, x)=$ $x_{1} \partial_{x_{j}} v_{t}(0, x)$ are in the space $H^{s-5}\left(\mathbf{R}^{n}\right)$. Also $x_{1} \partial_{x_{j}} v(0, x) \in H^{s-4}\left(\mathbf{R}^{n}\right)$ by the hypothesis. Suppose that the lemma is true for $k-1$. Note that $\left(x_{1} \partial_{x_{1}}\right)^{k}$ is a linear combination of $x_{1} \partial_{x_{1}}, \cdots, x_{1}^{k} \partial_{x_{1}}^{k}$. Therefore $x_{1}^{k} \partial_{x_{1}}^{k}$ is a linear combination of $\left(x_{1} \partial_{x_{1}}\right), \cdots,\left(x_{1} \partial_{x_{1}}\right)^{k}$ and so $x_{1}^{k} \partial_{x_{1}}^{k} v(0, x) \in H^{s-4}\left(\mathbf{R}^{n}\right)$ and $x_{1}^{k} \partial_{x_{1}}^{l} v_{t}(0, x) \in H^{s-5}\left(\mathbf{R}^{n}\right)$, for $1 \leq l \leq k$, by the induction hypothesis.

For $l_{1}=1$, we want to examine the regularities of $\left(x_{1}^{k} \partial_{t} \partial_{x_{1}}^{k-1}\right) v(0, x)$ and $x_{1}^{k} \partial_{t} \partial_{x_{j}}^{k-1} v(0, x)$. Since $\partial_{x_{1}}\left(x_{1}^{k} \partial_{t} \partial_{x_{1}}^{k-1}\right) v(0, x)=k x_{1}^{k-1} \partial_{x_{1}}^{k-1} v_{t}(0, x)+$ $x_{1}^{k} \partial_{x_{1}}^{k} v_{t}(0, x)$ and $\partial_{x_{j}}\left(x_{1}^{k} \partial_{t} \partial_{x_{1}}^{k-1}\right) v(0, x)=x_{1}\left(x_{1}^{k-1} \partial_{x_{1}}^{k-1}\right)\left(\partial_{x_{j}}\right) v_{t}(0, x)$ for $j$ $>1,\left(x_{1}^{k} \partial_{t} \partial_{x_{1}}^{k-1}\right) v(0, x) \in H^{s-4}\left(\mathbf{R}^{n}\right)$. Similarly $\left(x_{1}^{k} \partial_{t} \partial_{x_{j}}^{k-1}\right) v(0, x)$ is in the space $H^{s-4}\left(\mathbf{R}^{n}\right)$ for $j>1$. For $l_{1}>1, \partial_{t}^{2}=P_{2}+\sum a_{i j}(D u) \partial_{x_{i}} \partial_{x_{j}}$ from (1.1) and so

$$
\begin{aligned}
x_{1}^{k} \partial_{t}^{k} v(0, x) & =x_{1}^{k} \partial_{t}^{2}\left(\partial_{t}^{k-2} v\right)(0, x) \\
& =x_{1}^{k} P_{2} V(0, x)+x_{1}^{k} \sum a_{i j}(D u)\left(\partial_{x_{i}} \partial_{x_{j}} V\right)(0, x) \\
& =x_{1}^{k} \tilde{g}(D V)(0, x)+x_{1}^{k} \sum a_{i j}(D u)\left(\partial_{x_{i}} \partial_{x_{j}} V\right)(0, x),
\end{aligned}
$$


where $V(t, x)=\partial_{t}^{k-2} v(t, x)$ and $P_{2} V=\tilde{g}(D V)$ given by differentiating $P_{2} v=g(D v)$ by $\partial_{t}^{k-2}$. Therefore, by the induction hypothesis and Schauder's lemma, $x_{1}^{k} \partial_{t}^{k} v(0, x) \in H^{s-4}\left(\mathbf{R}^{n}\right)$. Similarly,

$$
x_{1}^{k} \partial_{t}^{l_{1}} \partial_{x_{1}}^{l_{2}} v(0, x)=x_{1}^{k} \partial_{x_{1}}^{l_{2}} \partial_{t}^{2}\left(\partial_{t}^{l_{1}-2} v\right)(0, x)
$$

and so $x_{1}^{k} \partial_{t}^{l_{1}} \partial_{x_{1}}^{l_{2}} v(0, x) \in H^{s-4}\left(\mathbf{R}^{n}\right)$. This completes the proof of lemma.

Theorem 3.10. Let $P_{2}$ be as above and let $\Sigma_{0}$ and $\Sigma_{1}$ be characteristic hypersurfaces for $P_{2}$ in $t>0$ intersecting transversally in $t=0$, with $\Gamma=\Sigma_{0} \cap \Sigma_{1}$. Suppose that $\Sigma_{0}$ and $\Sigma_{1}$ are given locally by $x_{1}=\varphi^{0}\left(t, x^{\prime}\right)$ and $x_{1}=\varphi^{1}\left(t, x^{\prime}\right)$ respectively with $\psi_{t} \neq 0$, where $\psi=\varphi^{0}-\varphi^{1}$. Suppose that $u \in C\left(\mathbf{R}^{+} ; H_{\mathrm{loc}}^{s}\left(\mathbf{R}^{n}\right)\right) \cap$ $C^{1}\left(\mathbf{R}^{+} ; H_{\mathrm{loc}}^{s-1}\left(\mathbf{R}^{n}\right)\right), s>\frac{n}{2}+4$, and $u$ satisfies the equation

$$
P_{2}(t, x, u, D u, D) u=f(t, x, u, D u),
$$

where $f$ is a smooth function of its arguments. Suppose that $\Gamma$ is smooth. If $u(0, x)$ and $u_{t}(0, x)$ belong to $N^{s, \infty}(\Gamma)$, then we have:

(1) $\Sigma_{0}$ and $\Sigma_{1}$ are smooth,

(2) $u$ is smooth outside $\Sigma_{0} \cup \Sigma_{1}$,

(3) locally, $u \in N^{s, \infty}\left(\Sigma_{0}, \Sigma_{1}\right)$.

Proof. By finite propagation speed and an analysis local in time, it may be assumed that $u$ has compact support in $x$ for each $t$. From now on, as a matter of notational convenience, we use $\varphi$ for both $\varphi^{0}$ and $\varphi^{1}$. As we see in the proof of Theorem 3.2, $\varphi \in C^{2}\left(\mathbf{R}^{+} \times \mathbf{R}^{n-1}\right)$ and for $s_{0}=s-2$,

$$
\varphi\left(t, x^{\prime}\right) \in C\left(\mathbf{R}^{+} ; H^{s_{0}}\left(\mathbf{R}^{n-1}\right)\right)
$$

since $s>\frac{n}{2}+4$.

Let $U$ stand for the vector of all derivatives of $u$ up to fourth order. By a smooth change of variables, we may assume that $\Gamma=\left\{x_{1}=0\right\}$. Then the vector fields tangential to $\Gamma$ are generated by $x_{1} \frac{\partial}{\partial x_{1}}, \frac{\partial}{\partial x_{2}}, \cdots, \frac{\partial}{\partial x_{n}}$.

Let $M$ stand for the vector operator $\left(1, M_{0}, M_{1}, \cdots, M_{n}\right)$. We note that $\left[M_{i}, D\right]=h_{i}\left(D^{2} \varphi, D\right)$. From (3.19),

$$
P_{2}(t, x, u, D u, D) M U=P_{1}(D) M U+G(D U),
$$

where $G(D U)=G\left(D U, D^{3} \varphi\right)$ and $c(D)=c\left(D^{3} u, D^{2} \varphi, D\right), d(D)=$ $d\left(D^{3} u, D^{3} \varphi, D\right)$ and $P_{1}(D)=P_{1}\left(D U, D^{2} \varphi, D\right)$ are first order operators.

The coefficients of $P_{2}$ are in $C\left(\mathbf{R}^{+} ; H^{s-1}\left(\mathbf{R}^{n}\right)\right) \cap C^{1}\left(\mathbf{R}^{+} ; H^{s-2}\left(\mathbf{R}^{n}\right)\right)$, while by (3.28) and (2.22), $G(D U)$ and the coefficients of $P_{1}\left(D U, D^{2} \varphi, D\right)$ are in the space $C\left(\mathbf{R}^{+} ; H^{s-5}\left(\mathbf{R}^{n}\right)\right)$.

Since $u(0, x)$ and $u_{t}(0, x)$ are conormal with respect to $\Gamma$, we have

$$
\begin{aligned}
& \left(x_{1} \partial_{x_{1}}\right)^{k_{1}}\left(\partial_{x_{2}}\right)^{k_{2}} \cdots\left(\partial_{x_{n}}\right)^{k_{n}} U(0, x) \in H^{s-4}\left(\mathbf{R}^{n}\right), \\
& \left(x_{1} \partial_{x_{1}}\right)^{k_{1}}\left(\partial_{x_{2}}\right)^{k_{2}} \cdots\left(\partial_{x_{n}}\right)^{k_{n}} U_{t}(0, x) \in H^{s-5}\left(\mathbf{R}^{n}\right) .
\end{aligned}
$$

Since $\varphi^{0}\left(0, x^{\prime}\right) \equiv 0 \equiv \varphi^{1}\left(0, x^{\prime}\right)$, by Taylor's Theorem, for $i=0$ and 1 ,

$$
\varphi^{i}(0, x)=t \int_{0}^{1} \varphi_{1}^{i}\left(s t, x^{\prime}\right) d s \equiv t \tilde{\varphi}^{i}\left(t, x^{\prime}\right),
$$

where $\varphi_{1}^{i}\left(t, x^{\prime}\right)=\frac{\partial \varphi^{i}}{\partial t}\left(t, x^{\prime}\right)$. 
Let $\tilde{\psi}=\tilde{\varphi}^{0}-\tilde{\varphi}^{1}$. Then $\psi=t \tilde{\psi}$. We note that $\varphi_{t}^{i}=\tilde{\varphi}^{i}+t \tilde{\varphi}_{t}^{i}, \varphi_{x_{j}}^{i}=$ $t \tilde{\varphi}_{x_{j}}^{i}, \psi_{x_{j}}=t \tilde{\psi}_{x_{j}}$ and

$$
\phi_{j}=\varphi_{t}^{0} \varphi_{x_{j}}^{1}-\varphi_{t}^{1} \varphi_{x_{j}}^{0}=t\left(\tilde{\varphi}^{0} \tilde{\varphi}_{x_{j}}^{1}-t \tilde{\varphi}_{t}^{0} \tilde{\varphi}_{x_{j}}^{1}-\tilde{\varphi}^{1} \tilde{\varphi}_{x_{j}}^{0}-t \tilde{\varphi}_{t}^{1} \tilde{\varphi}_{x_{j}}^{0}\right) .
$$

Therefore, $\phi_{j}\left(0, x^{\prime}\right) \equiv 0$, and $\psi_{x_{j}}\left(0, x^{\prime}\right) \equiv 0$.

We want to examine the regularity of $M U(0, x)$ by using the conormal regularity of the initial data. First, from (3.13)

$$
\begin{aligned}
\left(M_{0} U\right)(0, x) & =-\left(x_{1}-\varphi^{0}\left(0, x^{\prime}\right)\right) \frac{1}{\psi_{t}}\left(0, x^{\prime}\right)\left(\partial_{t}+\varphi_{t}^{1}\left(0, x^{\prime}\right) \partial_{x_{1}}\right) U(0, x) \\
& =-\frac{1}{\psi_{t}}\left(0, x^{\prime}\right)\left(x_{1} \partial_{t}\right) U(0, x)-\varphi_{t}^{1}\left(0, x^{\prime}\right)\left(x_{1} \partial_{x_{1}}\right) U(0, x) .
\end{aligned}
$$

Since $\partial_{x_{1}}\left(x_{1} \partial_{t}\right) U(0, x)=U_{t}(0, x)+\left(x_{1} \partial_{x_{1}}\right) U_{t}(0, x) \in H^{s-5}\left(\mathbf{R}^{n}\right)$ and $\partial_{x_{j}}\left(x_{1} \partial_{t}\right)$. $U(0, x)=\left(x_{1} \partial_{x_{j}}\right) U_{t}(0, x) \in H^{s-5}\left(\mathbf{R}^{n}\right),\left(x_{1} \partial_{t}\right) U(0, x) \in H^{s-4}\left(\mathbf{R}^{n}\right)$ and so $\left(M_{0} U\right)(0, x) \in H^{s-4}\left(\mathbf{R}^{n}\right)$.

Second,

$$
\begin{aligned}
\left(M_{1} U\right)(0, x) & =\left(x_{1}-\varphi^{1}\left(0, x^{\prime}\right)\right) \frac{1}{\psi_{t}}\left(0, x^{\prime}\right)\left(\partial_{t}+\varphi_{t}^{0}\left(0, x^{\prime}\right) \partial_{x_{1}}\right) U(0, x) \\
= & \frac{1}{\psi_{t}}\left(0, x^{\prime}\right)\left(x_{1} \partial_{t}\right) U(0, x)+\frac{1}{\psi_{t}}\left(0, x^{\prime}\right) \varphi_{t}^{0}\left(0, x^{\prime}\right)\left(x_{1} \partial_{x_{1}}\right) U(0, x) .
\end{aligned}
$$

Therefore $\left(M_{1} U\right)(0, x) \in H^{s-4}\left(\mathbf{R}^{n}\right)$. Finally, for $j=2, \cdots, n$,

$$
\begin{aligned}
& \left(M_{j} U\right)(0, x) \\
& \quad=\frac{1}{\psi_{t}}\left(0, x^{\prime}\right)\left(-\psi_{j}\left(0, x^{\prime}\right) \partial_{t} U(0, x)+\phi_{j} \partial_{x_{1}} U(0, x)+\psi_{t} \partial_{x_{j}}\right) U(0, x) \\
& \quad=\partial_{x_{j}} U(0, x),
\end{aligned}
$$

and so $\left(M_{j} U\right)(0, x) \in H^{s-4}\left(\mathbf{R}^{n}\right)$.

It then follows from Corollary 2.6 that

$$
M U \in C\left(\mathbf{R}^{+} ; H^{s-4}\left(\mathbf{R}^{n}\right)\right) \cap C^{1}\left(\mathbf{R}^{+} ; H^{s-5}\left(\mathbf{R}^{n}\right)\right) .
$$

In order to use the improved regularity of $U$, it is convenient to differentiate (2.22) two times and then we get (3.21).

Let $\mathscr{L}^{0}$ and $\mathscr{L}^{1}$ be as in the proof of Theorem 3.8. As we see in the proof of Theorem 3.8, it follows from (3.22) and Corollary 2.12 that $\tilde{L} D^{2} \varphi^{i} \in$ $C\left(\mathbf{R}^{+} ; H^{s_{0}-2}\left(\mathbf{R}^{n-1}\right)\right)$ since $\tilde{L} D^{2} \varphi^{i}\left(0, x^{\prime}\right) \in H^{s_{0}-2}\left(\mathbf{R}^{n-1}\right)$.

Applying $M$ to equation (3.19), we obtain the equation

$$
P_{2}\left(M^{2} U\right)=P_{12}(D)\left(M^{2} U\right)+G_{2}\left(D M U, M D^{2} \varphi\right),
$$

with $P_{12}=P_{12}\left(D U, D^{2} \varphi\right)$ a first order operator.

From (3.22), $M D^{2} \varphi \in C\left(\mathbf{R}^{+} ; H^{s_{0}-2}\left(\mathbf{R}^{n-1}\right)\right)$ and then, by Corollary 2.12 and Lemma 3.9,

$$
M^{2} U(t, x) \in C\left(\mathbf{R}^{+} ; H^{s-4}\left(\mathbf{R}^{n}\right)\right) \cap C^{1}\left(\mathbf{R}^{+} ; H^{s-5}\left(\mathbf{R}^{n}\right)\right) .
$$


Applying $L \in \mathscr{L}^{i}$ to (3.22), we have the equation

$$
\begin{aligned}
\left(\tilde{L}^{2} D^{2} \varphi^{i}\right)_{t}= & F_{12}\left(D u\left(t, \varphi^{i}, x^{\prime}\right), \tilde{D} \varphi^{i}\right) \tilde{D}\left(\tilde{L}^{2} D^{2} \varphi^{i}\right) \\
& +F_{22}\left(L D^{2} u\left(t, \varphi^{i}, x^{\prime}\right), \tilde{L} D^{2} \varphi^{i}, D^{2} \psi\right)\left(\tilde{L}^{2} D^{2} \varphi^{i}\right) \\
& +F_{32}\left(L^{2} D^{3} u\left(t, \varphi^{i}, x^{\prime}\right), \tilde{L} D^{2} \varphi^{i}, \tilde{L} D^{2} \psi\right)
\end{aligned}
$$

A similar argument as above implies that $\tilde{L}^{2} D^{2} \varphi \in C\left(\mathbf{R}^{+} ; H^{s_{0}-2}\left(\mathbf{R}^{n-1}\right)\right)$.

Suppose inductively that equation (3.28) is improved to $\tilde{L}^{k} D^{2} \varphi^{i} \in$ $C\left(\mathbf{R}^{+} ; H^{s_{0}-2}\left(\mathbf{R}^{n-1}\right)\right)$ and (3.29) is improved to $M^{k+1} U \in C\left(\mathbf{R}^{+} ; H^{s-4}\left(\mathbf{R}^{n}\right)\right) \cap$ $C^{1}\left(\mathbf{R}^{+} ; H^{s-5}\left(\mathbf{R}^{n}\right)\right)$. From the analogue of $(3.30)$ for $\tilde{L}^{k+1} D^{2} \varphi^{i}$ and Corollary 2.12 , it follows that

$$
\varphi \in C\left(\mathbf{R}^{+} ; H^{s_{0}+k+1}\left(\mathbf{R}^{n-1}\right)\right) \cap \cdots \cap C^{k+1}\left(\mathbf{R}^{+} ; H^{s_{0}}\left(\mathbf{R}^{n-1}\right)\right),
$$

since $\partial_{t}=\left(\psi_{t} / \psi\right) \tilde{L}_{1}$ and $\partial_{x_{j}}=\left(\psi_{x_{j}} / \psi\right) \tilde{L}_{1}-\tilde{L}_{j}$. Then, an equation similar to (3.19) holds for $M^{k+2} U$ with coefficients depending smoothly on $D M^{k+1} U$ and $M^{k+1} D^{2} \varphi$, that is,

$$
\begin{aligned}
& P_{2}(t, x, u, D u, D) M^{k+2} U \\
& \quad=P_{1(k+2)}(D) M^{k+2} U+G_{k+2}\left(D M^{k+1} U, M^{k+1} D^{2} \varphi\right),
\end{aligned}
$$

where $P_{1(k+2)}(D)=P_{1(k+2)}\left(D U, D^{2} \varphi, D\right)$ is a first order operator. Consequently, (3.20) may be improved to

$$
M^{k+2} U \in C\left(\mathbf{R}^{+} ; H^{s-4}\left(\mathbf{R}^{n}\right)\right) \cap C^{1}\left(\mathbf{R}^{+} ; H^{s-5}\left(\mathbf{R}^{n}\right)\right) .
$$

Since an equation of the form (3.31) holds for $\tilde{L}^{k+2} D^{2} \varphi^{i}$, that is,

$$
\begin{aligned}
\left(\tilde{L}^{k+2} D^{2} \varphi^{i}\right)_{t}= & F_{1(k+2)}\left(D u\left(t, \varphi^{i}, x^{\prime}\right), \tilde{D} \varphi\right) \tilde{D}\left(\tilde{L}^{k+2} D^{2} \varphi^{i}\right) \\
& +F_{2(k+2)}\left(L^{k+1} D u\left(t, \varphi^{i}, x^{\prime}\right), \tilde{L} D \varphi^{i}, D^{2} \psi\right)\left(\tilde{L}^{k+2} D^{2} \varphi^{i}\right) \\
& +F_{3(k+2)}\left(L^{k+2} D^{3} u\left(t, \varphi^{i}, x^{\prime}\right), \tilde{L}^{k+1} D^{2} \varphi^{i}, \tilde{L}^{k+1} D^{2} \psi\right),
\end{aligned}
$$

it follows that (3.28) is then improved to $\varphi \in C\left(\mathbf{R}^{+} ; H^{s_{0}+k+2}\left(\mathbf{R}^{n-1}\right)\right)$. The induction step is complete. Therefore, the conormal regularity of $u$ is established.

\section{Piecewise smoothness}

In this section, we will consider the piecewise smoothness of a solution $u$ of the equation $P_{2}(t, x, u, D u, D) u=f(D u)$.

Definition 4.1. Suppose that an open set $\Omega$ in $\mathbf{R}^{n+1}$ is divided into finitely many open components, $\Omega_{i}$, by a family of $C\left(\mathbf{R} ; H_{\mathrm{loc}}^{\tau}\left(\mathbf{R}^{n}\right)\right) \cap C^{1}\left(\mathbf{R} ; H_{\mathrm{loc}}^{\tau-1}\left(\mathbf{R}^{n}\right)\right)$, $\tau>\frac{n}{2}$, hypersurfaces $H_{1}, \cdots, H_{N}$. A function $u(t, x)$ is called piecewise continuous if its restriction to each $\Omega_{i}$ has a continuous extention to $\bar{\Omega}_{i}$, written

$$
u \in C\left(\Omega \backslash H_{1}, \cdots, H_{N}\right) .
$$

When the $H_{i}$ are clear from the context we will just say that $u$ is piecewise continuous (p.c.). A function $u(t, x)$ is called piecewise smooth if $D^{\alpha} u$ is piecewise continuous for all $\alpha$, written

$$
u \in C^{\infty}\left(\Omega \backslash H_{1}, \cdots, H_{N}\right) \text {. }
$$


In this section, by $u$ being piecewise smooth we mean that $D^{\alpha} u$ is piecewise continuous for all $|\alpha| \geq k$, for some $k$. Let $\mathscr{O}$ be an open neighborhood of the origin and let $\mathscr{Q}_{0}=\mathscr{O} \cap\{t=0\}$. Let $\Sigma_{0}, \Sigma_{1}$ and $\Gamma$ be as in section 3.2. With a slightly changed time coordinate, we may assume, by strict hyperbolicity of $P_{2}$ and the hypothesis of Theorem 3.8 since $\Gamma$ is smooth, that $\Gamma$ divides $\mathscr{O}_{0}$ into two connected components and $\Sigma_{0} \cup \Sigma_{1}$ divides $\mathscr{O}$ in four connected components.

In order to treat piecewise smoothness, we treat the initial value problem, as in Rauch-Reed [15], instead of making assumptions in $\{t<0\}$.

Theorem 4.1. Let $P_{2}$ be as in (1.2) and let $\Sigma_{0}$ and $\Sigma_{1}$ be characteristic hypersurfaces for $P_{2}$ intersecting transversally in $\{t=0\}$ with $\Gamma=\Sigma_{0} \cap \Sigma_{1}$. Suppose that $u \in C\left(\mathbf{R} ; H_{\mathrm{loc}}^{s}\left(\mathbf{R}^{n}\right)\right) \cap C^{1}\left(\mathbf{R} ; H_{\mathrm{loc}}^{s-1}\left(\mathbf{R}^{n}\right)\right), s>\frac{n}{2}+4$, and $u$ satisfies the equation

$$
P_{2}(t, x, u, D u, D) u=f(t, x, u, D u),
$$

where $f$ is a smooth function of its arguments. Suppose that $\Gamma$ is smooth. Suppose that $u(0, x) \in H_{\mathrm{loc}}^{s}\left(\mathbf{R}^{n}\right)$ and $u_{t}(0, x) \in H_{\mathrm{loc}}^{s-1}\left(\mathbf{R}^{n}\right)$ and $u(0, x), u_{t}(0, x) \in$ $C^{\infty}\left(\mathscr{O}_{0} \backslash \Gamma\right)$. Then $u(t, x) \in C^{\infty}\left(\mathscr{O} \backslash \Sigma_{0}, \Sigma_{1}\right)$.

Proof. We remark that the same argument as in section 3.2, except for the initial value problem instead of the case in which the hypotheses are made in $\{t<0\}$, implies that $\Sigma_{0}$ and $\Sigma_{1}$ are smooth away from $\Gamma$ and $u \in N^{s, \infty}\left(\Sigma_{0}, \Sigma_{1}\right)$. Therefore $u$ is smooth outside $\Sigma_{0} \cup \Sigma_{1}$.

It suffices to prove the theorem locally. We use $\varphi, D_{0}, D_{1}$ and $M_{j}$ as in the proof of Theorem 3.8. We will show for each $m \geq 0, i+j=m$ and for all $\alpha$, that

$$
D_{0}^{i} D_{1}^{j} M^{\alpha} v, D_{0}^{i} D_{1}^{j} M^{\alpha} w \in p . c .
$$

where $\alpha=\left(\alpha_{0}, \alpha_{1}, \cdots, \alpha_{n}\right), v=D_{0}\left(D^{3} u\right), w=D_{1}\left(D^{3} u\right)$ and $M$ is the vector operator $\left(M_{0}, M_{1}, \cdots, M_{n}\right)$. This will imply that the functions $\varphi^{0}$ and $\varphi^{1}$ are themselves piecewise smooth and hence the vector fields have piecewise smooth coefficients. Since $D_{0}, D_{1}$ and $\tilde{M}=\left(M_{2}, \cdots, M_{n}\right)$ span $T\left(\mathbf{R} \times \mathbf{R}^{n}\right)$, this implies the desired result.

The proof is basically by induction on $m$. Recall from Theorem 3.8 that $M^{\beta} D^{3} u \in C\left(\mathbf{R} ; H_{\operatorname{loc}}^{s-3}\left(\mathbf{R}^{n}\right)\right) \cap C^{1}\left(\mathbf{R} ; H_{\text {loc }}^{s-4}\left(\mathbf{R}^{n}\right)\right), M^{\beta}\left(D^{2} \varphi\right) \in C\left(\mathbf{R} ; H_{\text {loc }}^{s-4}\left(\mathbf{R}^{n-1}\right)\right)$ for all $\beta$. For $m=0$ and $\alpha=0$, since $s>\frac{n}{2}+4, \varphi\left(t, x^{\prime}\right) \in C^{2}\left(\mathbf{R} \times \mathbf{R}^{n-1}\right)$ and so $v, w \in C\left(\mathbf{R} \times \mathbf{R}^{n}\right)$. Suppose that $M^{\alpha} v, M^{\alpha} w \in C\left(\mathbf{R} \times \mathbf{R}^{n}\right)$ for $|\alpha|=k-1$. Then

$$
M_{j} M^{\alpha} v=D_{0} M_{j} M^{\alpha} D^{3} u+\left[M_{j} M^{\alpha}, D_{0}\right] D^{3} u \in C\left(\mathbf{R} \times \mathbf{R}^{n}\right)
$$

By Lemma 3.4, $\left[M_{0}^{k}, D_{0}\right]=\sum_{j=0}^{k-1} c_{j}(k) D_{0} M_{0}^{j}$, where $c_{j}(k)$ are constants depending only on $k$. Therefore, $M^{\alpha} v \in C\left(\mathbf{R} \times \mathbf{R}^{n}\right)$ for $|\alpha|=k$. Similarly, $M^{\alpha} w \in C\left(\mathbf{R} \times \mathbf{R}^{n}\right)$.

In order to see the induction process more precisely, we prove (4.2) for $m=$ 1. From (3.15) and the equation $P_{2}\left(D^{3} u\right)=G\left(D D^{3} u\right), v$ and $w$ satisfy 
equations of the form:

$$
\begin{aligned}
D_{0} w & +r_{00} M_{0} v+r_{11} M_{1} w+\sum_{j=2}^{n} r_{0 j} M_{j} v+\sum_{j=2}^{n} r_{1 j} M_{j} w+\sum_{i, j=2}^{n} r_{i j} M_{i} M_{j} D^{3} u \\
& =f\left(D^{2} u, v, w, M D^{3} u, D^{2} \varphi\right)
\end{aligned}
$$

and

$$
\begin{aligned}
D_{1} v & +r_{00} M_{0} v+r_{11} M_{1} w+\sum_{j=2}^{n} r_{0 j} M_{j} v+\sum_{j=2}^{n} r_{1 j} M_{j} w+\sum_{i, j=2}^{n} r_{i j} M_{i} M_{j} D^{3} u \\
& =f\left(D^{2} u, v, w, M D^{3} u, D^{2} \varphi\right),
\end{aligned}
$$

where $f$ is a smooth function of its arguments. Therefore, by the case of $m=0, D_{0} w$ and $D_{1} v$ are in the space $C\left(\mathbf{R} \times \mathbf{R}^{n}\right)$.

We apply $M_{i}$ to equations (4.4) and (4.5). Then we have

$$
\begin{aligned}
M_{i} D_{0} w= & M_{i} D_{1} v \\
= & \left.M_{i} r_{00}\right) M_{0} v+r_{00} M_{i} M_{0} v+\left(M_{i} r_{11}\right) M_{1} w+r_{11} M_{i} M_{1} w \\
& +\sum_{j}\left(M_{i} r_{0 j}\right) M_{j} v+r_{0 j} M_{i} M_{j} v+\sum_{j}\left(M_{i} r_{1 j}\right) M_{j} w+r_{1 j} M_{i} M_{j} w \\
& +\sum_{j, k}\left(M_{i} r_{j k}\right) M_{j} M_{k} D^{3} u+r_{j k} M_{i} M_{j} M_{k} D^{3} u \\
& +g\left(M_{i} D^{2} u, M_{i} v, M_{i} w, M_{i} M D^{3} u, M_{i} D^{2} \varphi\right),
\end{aligned}
$$

where $g$ is a smooth function of its arguments. Therefore, by (4.3) and since $M_{i} D^{2} \varphi \in C\left(\mathbf{R} ; H^{s-4}\left(\mathbf{R}^{n}\right)\right)$, we have $M_{i} D_{1} v, M_{i} D_{0} w \in C\left(\mathbf{R} \times \mathbf{R}^{n}\right)$ for all $i=0,1, \cdots, n$. By an induction argument and improved piecewise regularity of $\varphi$ with respect to $M_{i}$, we have

$$
M^{\alpha} D_{1} v, M^{\alpha} D_{0} w \in C\left(\mathbf{R} \times \mathbf{R}^{n}\right) \text { for all } \alpha .
$$

In order to show that $D_{0} v$ is piecewise continuous, we apply $D_{0}$ to equation (4.5) to obtain the equation

$$
\begin{aligned}
D_{1}\left(D_{0} v\right) & +\left(D_{0} r_{00}\right) M_{0} v+r_{00} M_{0} D_{0} v+\left(D_{0} r_{11}\right) M_{1} w+r_{11} M_{1} D_{0} w \\
& +\sum_{j}\left(D_{0} r_{0 j}\right) M_{j} v+r_{0 j} M_{j} D_{0} v+\sum_{j}\left(D_{0} r_{1 j}\right) M_{j} w+r_{1 j} M_{j} D_{0} w \\
& +\sum_{j, k}\left(D_{0} r_{j k}\right) M_{j} M_{k} D^{3} u+r_{j k} M_{j} M_{k} D_{0} D^{3} u \\
= & f_{1}\left(D_{0} D^{2} u, D_{0} v, D_{0} w, M D_{0} D^{3} u, D_{0} D^{2} \varphi\right),
\end{aligned}
$$

or

$$
Q_{+}\left(D_{0} v\right)+g_{0}\left(D^{2} u, v, w, M D^{3} u, D^{2} \varphi\right)\left(D_{0} v\right)=g_{1},
$$

where $Q_{+}=D_{1}+r_{00} M_{0}+\sum_{j=2}^{n} r_{0 j} M_{j}, g_{0}$ is a smooth function of its arguments and $g_{1}$ is a smooth function of its arguments $D_{0} D^{2} u, D_{0} D^{2} \varphi, M_{0} v, M_{1} w$, $M_{1} D_{0} w, M_{j} v, M_{j} w, M_{j} D_{0} w, M_{i} M_{j} D^{3} u$ and $M_{i} M_{j} v$. By applying $D_{0}$ to 
equation (3.21), we have the equation of form (3.22) replaced $L$ and $\tilde{L}$ by $D_{i}$ and $\frac{1}{\psi_{t}} \partial_{t}$. Therefore, we have

$$
Q\left(D_{0} D^{2} \varphi^{1}\right)=G_{1}\left(D_{0} D^{2} \varphi^{1}\right)+G_{2},
$$

where $Q=\partial_{t}-f^{\prime}\left(D u\left(t, \varphi, x^{\prime}\right), \tilde{D} \varphi\right) \sum_{j=2}^{n} \partial_{x_{j}}$ and $G_{1}, G_{2}$ are smooth functions of their arguments. Since $Q$ is transversal to $\Sigma_{0}, \Sigma_{1}$ and $\{t=0\}$ by the slightly changed time coordinate, $D_{0} D^{2} \varphi$ is piecewise continuous by Lemma 4.2. Here we use the fact that piecewise continuous functions form an algebra invariant under composition with $C^{\infty}$ functions. Therefore, (4.6) can be written as

$$
Q_{+}\left(D_{0} v\right)+(\text { p.c. })\left(D_{0} v\right)=\text { p.c. }
$$

We note that $Q_{+}$is a vector field transverse to $\Sigma_{1}$ and $t=0$. Since $t=0$ is a noncharacteristic hypersurface, all derivatives may be computed from the initial data at $t=0$ and thus $D_{0} v(0, x)$ is p.c. Therefore, by Lemma 4.2, $D_{0} v$ is p.c. on $\mathscr{O} \backslash \Sigma_{0}$. A similar proof for $Q_{-}=D_{0}+r_{11} M_{1}+\sum_{j=2}^{n} r_{1 j} M_{j}$ shows that $D_{1} w$ is p.c. on $\mathscr{O} \backslash \Sigma_{1}$.

In order to show that $D_{0} M_{i} v$ and $D_{1} M_{i} w$ are p.c. for $i=0,1, \cdots, n$, we differentiate (4.6) with respect to $M_{i}$. Then we have

$$
Q_{+}\left(M_{i} D_{0} v\right)+\left(h_{0}\right)\left(M_{i} D_{0} v\right)=\left(h_{1}\right),
$$

where $h_{0}$ and $h_{1}$ are smooth functions of their arguments, which are all p.c. Note that $D_{0} M_{i} D^{2} \varphi$ is piecewise continuous by similar arguments as above after applying $M_{i}$ to equation (4.7). $D_{0} M_{i} v$ satifies an initial value problem of the form (4.11), and then, by Lemma 4.2, $D_{0} M_{i} v$ is p.c. on $\mathscr{O} \backslash \Sigma_{0}$. A similar proof for $Q_{-}=D_{0}+r_{11} M_{1}+\sum_{j=2}^{n} r_{1 j} M_{j}$ shows that $D_{1} M_{i} w$ is p.c. on $\mathscr{O} \backslash \Sigma_{1}$. Again, by an induction argument and improved piecewise regularity of $\varphi$ with respect to $D_{0}, D_{1}$ and $M_{i}$, we have

$$
M^{\alpha} D_{0} v, M^{\alpha} D_{1} w \in C\left(\mathbf{R} \times \mathbf{R}^{n}\right) \quad \text { for all } \alpha .
$$

We assume that (4.2) and $D_{0}^{i} D_{1}^{j} M^{\alpha} v, D_{0}^{i} D_{1}^{j} M^{\alpha} w$, hold for $i+j=m-1$. Then we will show that (4.2) holds for $m$. Using the induction hypothesis, it follows from equations (4.4) and (4.5) that

$$
\begin{gathered}
D_{0}^{i} D_{1}^{j} M^{\alpha} w \in \text { p.c. } \quad \text { if } i \geq 1, \\
D_{0}^{i} D_{1}^{j} M^{\alpha} v \in \text { p.c. } \quad \text { if } j \geq 1 .
\end{gathered}
$$

Therefore, it remains to show that $D_{0}^{m} M^{\alpha} v$ and $D_{1}^{m} M^{\alpha} w$ are p.c. We will work on the $v$ terms: the $w$ terms are treated similarly.

By applying $D_{0}^{m-1}$ to (4.6) and using the induction hypothesis, we obtain an equation

$$
Q_{+}\left(D_{0}^{m} v\right)+(\text { p.c. })\left(D_{0}^{m} v\right)=\text { p.c. }
$$

Therefore, by a similar argument to that given in the case $m=1, D_{0}^{m} v$ is p.c. on $\mathscr{O} \backslash \Sigma_{0}$. A similar proof for $Q_{-}$shows that $D_{1}^{m} w$ is p.c. on $\mathscr{O} \backslash \Sigma_{1}$. This completes the proof of (4.2) for $\alpha=0$.

In order to consider the case $|\alpha|=1$, we differentiate (4.10) with respect to $M_{i}$ noting that the p.c. terms remain p.c. Note that by the arguments as above 
piecewise regularity of $\varphi$ with respect to $D_{0}$ and $M_{i}$ is inductively improved. Then we have

$$
Q_{+}\left(M_{i} D_{0}^{m} v\right)+\left(H_{0}\right)\left(M_{i} D_{0}^{m} v\right)=H_{1} .
$$

$M_{i} D_{0}^{m} v$ satisfies an initial value problem of the form (4.11). Therefore, by Lemma 4.2, we can conclude that $D_{0}^{m} M_{i} v$ is p.c. on $\mathscr{O} \backslash \Sigma_{0}$. Similarly, $D_{1}^{m} M_{i} w$ is p.c. on $\mathscr{O} \backslash \Sigma_{1}$ and we have proven (4.2) in the case $|\alpha|=1$. The general case is proved in the same method by induction on $|\alpha|$. The proof of the theorem is completed, except for Lemma 4.2.

Lemma 4.2. Suppose that $A(t, x)$ and $F(t, x)$ are p.c. on $\mathscr{O} \backslash \Sigma_{0} \cup \Sigma_{1}$. Then the initial value problem for the linear equation

$$
Q_{+} z(t, x)+A(t, x) z(t, x)=F(t, x)
$$

has a unique piecewise continuous solution on $\mathscr{O} \backslash \Sigma_{0}$ if $z(0, x)$ is piecewise continuous on $\mathscr{O}_{0} \backslash \Gamma$. Here $Q_{+}$is a first order operator with piecewise continuous coefficients as described after (4.6).

Proof. The argument is similar to that in Rauch and Reed [15] except that the coefficients of $Q_{+}$are merely assumed to be piecewise continuous instead of smooth.

I thank Professor Michael Beals for help in completing this paper. Most of this work is my Ph.D thesis, and the rest was written while in communication with him.

\section{REFERENCES}

1. S. Alinhac, Paracomposition et application aux equations nonlineaires, Bony-SjöstrandMeyer, no. 11 (1984-1985), École Polytechnique, Paris.

2. Interaction d'ondes simple pour des équations complétement nonlineaires, Sém. d'E.D.P. no. 8 (1985-1986), École Polytechnique, Paris.

3. M. Beals, Self-spreading and strength of singularities for solutions to semilinear wave equation, Ann. of Math. (2) 118 (1983), 187-214.

4. _ Propagation and interaction of singularities in nonlinear hyperbolic problems, Birkhäuser, Boston, 1989.

5. M. Beals and M. Reed, Propagation of singularities for hyperbolic pseudo differential operators with nonsmooth coefficients, Comm. Pure Appl. Math. 35 (1982), 169-184.

6. __ Microlocal regularity theorems for nonsmooth pseudo-diffrential operators and applications to nonlinear problems, Trans. Amer. Math. Soc. 285 (1984), 159-184.

7. J. M. Bony, Interaction des singularités pour les équations aux dérivées partielles nonlinéaires, Sem. Goulaouic-Meyer-Schwartz 22 (1979-80).

8. Interaction des singularités pour les équations aux dérivées partielles non-linéaires, Sem. Goulaouic-Meyer-Schwartz 2 (1981-82).

9. T. Messer, Propagation of singularities of hyperbolic systems, Indiana Univ. Math. J. 36 (1987), 45-77.

10. Y. Meyer, Remarques sur un theoreme de J. M. Bony, Suppl. Rend. Circ. Mat. Palermo 1 (1981), 1-20.

11. L. Nirenberg, On elliptic partial differential equations, Ann. Scuola Norm. Sup. Pisa. 13 (1959), 117-162.

12. L Lecture on linear partial differential equation, C.B.M.S. Regional Conf. Ser. in Math., no. 17, Amer. Math. Soc., Providence, RI, 1973.

13. J. Rauch, Singularities of solutions to semilinear wave equations, J. Math. Pures Appl. 58 (1979), 299-308. 
14. J. Rauch and M. Reed, Propagation of singularities for semilinear hyperbolic equations in one space variable, Ann. of Math. 111 (1980), 531-552.

15. _. Striated solutions of semilinear, two-speed wave equations, Indiana Univ. Math. J. 34 (1985), 337-353.

Department of Mathematics, Duksung Women's University, 132-714 Seoul, Korea E-mail address: sjkang@namhae.duksung. $\mathrm{kr}$ 